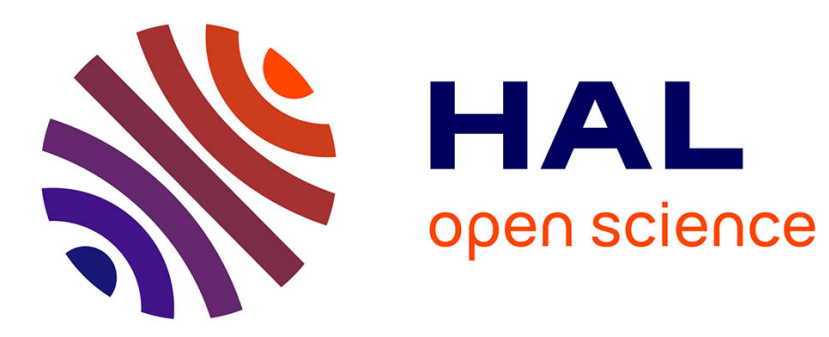

\title{
The volcano-electric effect
}

\author{
A Revil, G Saracco, P Labazuy
}

\section{To cite this version:}

A Revil, G Saracco, P Labazuy. The volcano-electric effect. Journal of Geophysical Research: Solid Earth, 2003, 108 (B5), 10.1029/2002JB001835 . hal-01760068

\section{HAL Id: hal-01760068 https://hal.science/hal-01760068}

Submitted on 9 Feb 2021

HAL is a multi-disciplinary open access archive for the deposit and dissemination of scientific research documents, whether they are published or not. The documents may come from teaching and research institutions in France or abroad, or from public or private research centers.
L'archive ouverte pluridisciplinaire HAL, est destinée au dépôt et à la diffusion de documents scientifiques de niveau recherche, publiés ou non, émanant des établissements d'enseignement et de recherche français ou étrangers, des laboratoires publics ou privés. 


\title{
The volcano-electric effect
}

\author{
A. Revil and G. Saracco \\ Department of Hydrogeophysics and Porous Media, CNRS-Centre Européen de Recherche et d'Enseignement \\ des Géosciences de l'Environment, Aix-en-Provence, France \\ P. Labazuy \\ Laboratoire Magmas et Volcans, Université Blaise Pascal, Clermont-Ferrand, France
}

Received 18 February 2002; revised 6 August 2002; accepted 27 February 2003; published 15 May 2003.

[1] The formation of a magmatic intrusion at depth is responsible for the formation of various thermohydromechanical (THM) disturbances including the upsurge of shock waves and diffusion of pressure fronts in the volcanic system. We couple electromagnetic theory (Maxwell equations) and thermoporoelasticity (Biot equations) to look at the ground surface electrical signature of these THM disturbances. The nature of this coupling is electrokinetic, i.e., associated with water flow relative to the mineral framework and the drag of the excess of charge located in the vicinity of the pore water/mineral interface (the groundwater flow disturbance being related here to the THM disturbances in drained conditions). A new set of laboratory data shows that the electrokinetic coupling is very substantial in fractured basaltic and volcaniclastic materials, and in scoria with several hundreds of millivolts of electrical potential gradient produced per megapascal of pore fluid pressure variations. Our theoretical analysis predicts the diffusion of electromagnetic disturbances and quasi-static electrical signals. These signals can be used as precursors of a volcanic eruption. Indeed, electromagnetic phenomena recorded at the ground surface of a volcanic system, once properly filtered to remove external contributions, provide a direct and quasi-instantaneous insight into the THM disturbances occurring in the heart of the volcanic structure prior and during a volcanic event. Tomography of the quasi-static electrical field is discussed and applied to self-potential profiles performed at the Piton de la Fournaise volcano during the preparation phase of the March 1998 eruption. INDEX TERMS: 0925 Exploration Geophysics: Magnetic and electrical methods; 1832 Hydrology: Groundwater transport; 5109 Physical Properties of Rocks: Magnetic and electrical properties; 5114 Physical Properties of Rocks: Permeability and porosity; 5139 Physical Properties of Rocks: Transport properties; KEYWORDS: self-potential, geoelectric, forecasting, volcanic activity, tomography, shock wave

Citation: Revil, A., G. Saracco, and P. Labazuy, The volcano-electric effect, J. Geophys. Res., 108(B5), 2251, doi:10.1029/2002JB001835, 2003.

\section{Introduction}

[2] Early diagnosis of volcanic eruptions is a difficult task that has led geophysicists and volcanologists to measure various kinds of signals at the ground surface of active volcanoes. Among these signals, time and space electromagnetic disturbances of relatively high amplitudes (several tens of nanotesla for the magnetic field and several hundreds of millivolts for the electrical potential) have been clearly evidenced in a substantial number of field studies and correlated with volcanic activity [Johnston and Stacey, 1969; Zlotnicki and Le Mouël, 1988; Sasai et al., 1990; Tanaka, 1993; Di Maio and Patella, 1994; Hashimoto and Tanaka, 1995; Malengreau et al., 1994; Del Negro et al., 1997; Di Maio et al., 1996, 1998; Michel and Zlotnicki, 1998].

Copyright 2003 by the American Geophysical Union. 0148-0227/03/2002JB001835\$09.00
[3] Active volcanic systems are also subject to thermohydromechanical (THM) disturbances in the preparation phase of a volcanic event. Several types of THM effects can coexist in a volcanic system. For example, Merlani et al. [2001] investigated a model of rock fracturing in the subsurface of hydrothermal systems in response to temperature and pore fluid pressure perturbations. In such conditions, thermoporoelastic theory predicts the upsurge of hot and pressurized fluid fronts, which take the form of nonlinear thermomechanical and mechanical wave solutions of two Bürgers equations. These "solitary" shock waves are associated with an upsurge of pore water yielding hydrothermal brecciation of the porous rock during their ascent. The upsurge of pore water depends in turn on the pore fluid pressure gradient carried up with these waves. When these shock waves reach the ground surface, they generate paroxysmal fluid emissions. A set of observations made at Vulcano (Aeolian Islands, 1987-1989) and Campi Fregrei 
(Italy, 1982-1984) are qualitatively in agreement with the arrival of such hot and pressurized fluid fronts at the ground surface [e.g., Natale, 1998; Natale et al., 1998]. Other catastrophic hydrovolcanic phenomena have been described in the recent literature (see a short review by Fontaine et al. [2002]).

[4] The physical link between the two phenomena described above, that is between the electromagnetic theory (described by the Maxwell equations) and thermoporoelasticity (described by the Biot equations) can be found in the electrokinetic coupling [e.g., Haartsen et al., 1998]. Indeed, THM disturbances are responsible for forced groundwater flow in a volcanic system as shown recently by Matsumoto et al. [2002] for Usu volcano. The flow of groundwater inside a porous material generates an electrical field of electrokinetic nature directed in the flow direction [e.g., Bernabé, 1998]. This electrical field is the result of a polarization mechanism associated with the relative displacement between the solid grains and the pore fluid contained in the connected pore space of the porous material (see Ishido and Mizutani [1981] for a description of the underlying physics of this phenomenon). Therefore hydrogeological phenomena occurring in active volcanic areas are probably the source of the observed electrical signals. Magnetic signature of groundwater flow is investigated in a separate contribution.

[5] Clint [1999] and Yoshida [2001] performed laboratory mechanical tests using brine-saturated low-permeability basalt cores among different types of porous rocks. They showed that once stressed, a low-permeability basalt sample behaves first as a closed system generating localized electrical current flow during the initial stage of deformation. When permeability is increased through microcracking, they observed a strong increase of electrical potential variations at the end faces of the sample as fluid flow pathways become more and more continuous. They conclude that the electrokinetic effect was at the origin of their observed electrical signals. Note that other mechanisms related to volcanic eruption can produce very large, short time electrical potential anomalies. For example, Büttner et al. [2000] analyzed highly transient (few milliseconds) electrical potential signals $(>100 \mathrm{~V})$ resulting from thermohydrodynamic fragmentation of magma in the volcanic conduit at Stromboli volcano.

[6] In this paper, we provide an analysis of the various electrical signals of electrokinetic nature produced in an active volcano and associated with THM disturbances. These signals are described in a new theoretical framework named Self-Potential Investigations and Comprehensive Evaluation (SPICE).

\section{Definition of a Reference State}

[7] Before defining what could be an "electrical disturbance" prior to a volcanic event, it is legitimate to define a reference state in which a "normal" electrical potential distribution can be associated with a "normal" pattern of groundwater circulation.

\subsection{Electrokinetic Coupling}

[8] The Darcy velocity (hydraulic flux) is defined by $\mathbf{u} \equiv$ $\phi\left(\mathbf{V}_{f}-\mathbf{V}_{S}\right)$, where $\phi$ is the porosity of the porous rock, $\mathbf{V}_{f}$ and $\mathbf{V}_{S}$ represent the mean velocity of the pore fluid and mineral framework, respectively, in an Eulerian framework of reference. The Darcy velocity is the volume of water flowing per unit surface area and per unit time. Similarly, the electrical current density represents the amount of electrical charge moving per unit surface area and per unit time. The flow of pore water itself, even if it contains cations and anions, is not in principle a source of electrical signals. Indeed, the amount of cations balances the amount of anions (electroneutrality condition), and there is no net charge carried along with the flow of pore water. However, in porous materials, there is actually an excess of charge in the pore water due to chemical complexation of the active hydroxyl groups located at the mineral/water interface. This excess of charge takes the form of a diffuse layer of counterions located in the close vicinity of the mineral/ pore water interface. In such a case, the flow of the pore water generates a net source current density in the medium. The opposite effect (i.e., the flow of the pore water resulting from the application of an electrical field) is also observed in porous materials (electro-osmosis) for the same reason, that is due to the existence of an excess of free charge in the vicinity of the pore water/mineral interface and viscous coupling with the pore water in the NavierStokes equation.

[9] It results from previous considerations that the Darcy velocity $\mathbf{u}$ and the electrical current density $\mathbf{j}$ (in $\mathrm{A} \mathrm{m}^{-2}$ ) form two coupled linear equations in porous materials. The physics of this coupling is discussed by many authors [e.g., Bernabé, 1998 and Yoshida, 2001]. These equations can be partially decoupled, i.e., in most cases electro-osmosis can be neglected. Therefore the hydraulic problem can be treated first and its solution used to evaluate electromagnetic disturbances generated in the system, which can be, in turn, easily measured and used to provide constraints about the groundwater flow pattern. The total electrical current density $\mathbf{j}$ is given by:

$$
\mathbf{j}=\sigma \mathbf{E}-L\left(\nabla p-\rho_{f} \mathbf{g}\right)=\sigma \mathbf{E}+\mathbf{j}_{S}
$$

where $\mathbf{j}_{S}$ is a source current density, $\mathbf{g}$ is the gravity acceleration vector (in $\mathrm{m} \mathrm{s}^{-2}$ ), $\mathbf{E}$ is the electrical field, $p$ is the pore fluid pressure, $\sigma$ is the electrical conductivity of the porous rock, $\rho_{f}$ is the density of the pore water, and $L$ is an electrokinetic coupling term. Revil and Leroy [2001] provide an expression of $L$ in terms of both electrochemical properties of the mineral/water interface (zeta potential and surface conductivity) and textural parameters (porosity and grain shape) of the porous rock. The electrical current density $\mathbf{j}_{S}$ is termed the streaming current. Using the Darcy equation (B5), the streaming current $\mathbf{j}_{S}$ is proportional to the Darcy velocity:

$$
\mathbf{j}_{S}=\frac{L \phi \eta_{f}}{k}\left(\mathbf{V}_{f}-\mathbf{V}_{S}\right)=\frac{L \eta_{f}}{k} \mathbf{u}
$$

Therefore a net electrical current density can be associated with the flow of the groundwater. In turn, $\mathbf{j}_{S}$ serves as a source term in the Maxwell equations for the generation of electromagnetic disturbances as shown further in section 4.1. 


\subsection{Electrical Field Pattern in the Reference State}

[10] We consider a volcano in a quiescent state. In this case, two types of groundwater flow dominate. The first is related to convective effects associated with temperature gradient and fluid density variations (RayleighBénard free convection with an open boundary at the top surface and eventually on the sides). The second contribution is related to gravitational downflow of the groundwater in aquifers along the flanks of the volcano. This yields

$$
\mathbf{u}=\mathbf{u}_{0}+\mathbf{u}_{1},
$$

where $\mathbf{u}_{0}$ represents the contribution associated with piezometric head variations under hydrostatic conditions and $\mathbf{u}_{1}$ is related to convective effects [Revil et al., 1999]. So according to equations (2) and (3), the source current density can also be split into two terms:

$$
\mathbf{j}_{S}=\mathbf{j}_{0}+\mathbf{j}_{1}
$$

where $\mathbf{j}_{0}$ and $\mathbf{j}_{1}$ represent the two contributions associated with $\mathbf{u}_{0}$ and $\mathbf{u}_{1}$, respectively. In both cases, the electrokinetic effect represents a dipolar separation of charge at the local scale of a representative elementary volume. For an unconfined aquifer, the electrical potential distribution associated with the contribution $\mathbf{j}_{0}$ can be represented as a double layer of charge (electrical dipoles) lying on the piezometric surface [Fournier, 1989]. Revil et al. [2002a, 2002b] demonstrate that this double layer is simply related to the volume density of current dipole moment (current polarization) resulting from groundwater flow associated with piezometric head variations.

[11] The second distribution represents a volume distribution of dipoles mathematically similar to that described by Revil et al. [2001]. This second contribution can be used to model the self-potential positive anomalies associated with the upward migration of hot vapor/water through the hydrothermal system (e.g., the large positive anomaly shown in Figure 1). Indeed, the upward flow of water, confined within a column, produced a positive selfpotential signal at the top of the column with respect to an arbitrary distant point at the ground surface [Poldini, 1938]. This fact is very well explained by the electrokinetic theory both in terms of polarity and amplitude [Revil, 2002a].

[12] In the quasi-static limit of the Maxwell equations, it follows that the total contribution to the electrical potential at the observation point $P$ is the sum of all the contributions, hence

$$
\begin{aligned}
\varphi(P)= & \frac{C^{\prime}}{2 \pi} \int_{\partial \Omega}\left(h-h_{0}\right)\left(\frac{\mathbf{x} \cdot \mathbf{n}}{x^{3}}\right) d S+\frac{1}{2 \pi} \int_{\Omega} \frac{\rho \nabla \cdot \mathbf{j}_{1}}{x} d V \\
& +\frac{1}{2 \pi} \int_{\Omega} \frac{\mathbf{E}}{x} \cdot \frac{\nabla \rho}{\rho} d V,
\end{aligned}
$$

where $\mathbf{x} \equiv \mathbf{r}-\mathbf{r}^{\prime}$ and $x=|\mathbf{x}|$ represent the distance between the observation point $P$ and the infinitesimal volume element $d V$ of the conductive medium surrounding the
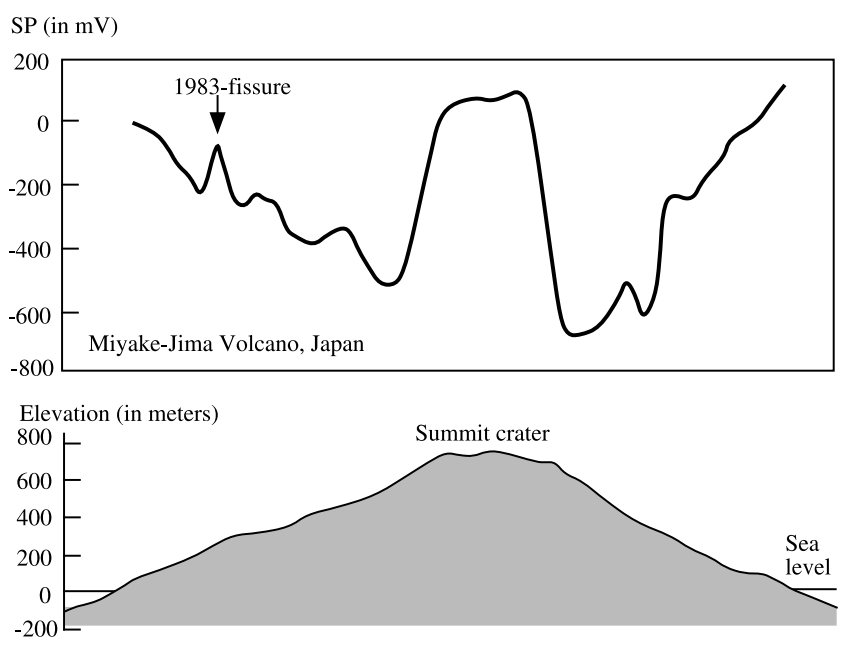

Figure 1. Typical self-potential distribution for a volcano in quiescent (reference) state (modified from the work of Sasai et al. [1990]). This self-potential pattern is the sum of a distribution associated with gravitational downflow of groundwater along the slopes of the volcano and a signal associated with hydrothermal groundwater flow, especially in the central part of the system. Prior to an eruptive event, we expect fluctuations of the electrical field, especially around the central crater, in response to THM disturbances associated with magma intrusion at depth.

integration point $M, \mathbf{n}$ is the outward normal to the water table, $d S$ is a surface element of the water table, $p_{0}$ is the background fluid pressure (reference level), $h$ is the piezometric head at the source point $M, h_{0}$ is the piezometric head at a reference level (e.g., the sea level), $\rho$ is here the electrical resistivity of the porous body, and $C^{\prime}$ represents an electrokinetic coupling coefficient related to the laboratory electrokinetic coupling coefficient $C$ (see section 5) by $C^{\prime}=$ $C \rho_{f}$ g. In equation (5), the first term corresponds to the contribution associated with piezometric head variation [Revil et al., 2002a], the second term corresponds to convective effects [Revil et al., 1999], and the third term corresponds to charge accumulation at surface or in volume where electrical resistivity gradients exist [Patella, 1997]. Equation (5) explains the self-potential distribution shown in Figure 1. The first contribution of equation (5) explains the electrical potential distribution on the flanks of the volcano associated with topography of the water table. The second contribution is associated with the hydrothermal system, which is usually well developed inside the central portion of the volcanic system.

[13] Next let us consider what happens when a volcanic system is perturbed from this reference state. In the preparation phase of a volcanic eruption, there are THM disturbances inside the volcanic system. These perturbations are the source of additional forced groundwater circulations, which generate transient electrical (electromagnetic) field disturbances by comparison with the reference level discussed above.

\section{THM Disturbances}

[14] In the preparation phase of an eruptive event, several types of THM disturbances occur in the volcanic system. In 
turn, these THM disturbances are responsible for disturbances of the flow of the groundwater by comparison with that observed in the reference state. In addition to these THM disturbances, the flow of the groundwater is also perturbed by an increase of the hydrothermal convection in the system, which can become, under some conditions, turbulent [e.g., Fontaine et al., 2002]. We first discuss in this section, three possible types of THM disturbances.

\subsection{Nonpropagative THM Disturbances (Type I)}

[15] The first class of THM phenomena corresponds to nonpropagative disturbances (noted Type I below). In thermoporoelastic materials, conservation of energy and momentum leads to two coupled equations, which govern the variations of the pore fluid pressure $p$ and temperature $T$ with time $t$ in the water-saturated porous system (Appendix A):

$$
\begin{gathered}
\nabla \cdot\left(\eta_{H} \nabla p\right)=-\frac{R}{H} \frac{d \sigma}{d t}-R \alpha_{m} \frac{d T}{d t}+\frac{d p}{d t}, \\
\frac{\partial T}{\partial t}-\eta_{T} \nabla^{2} T-\beta_{T} \nabla p \cdot \nabla T=0, \\
\eta_{H} \equiv \frac{k R}{\eta_{f}}, \\
\eta_{T} \equiv \frac{\lambda}{\rho c}, \\
\beta_{T} \equiv \frac{\rho_{f} c_{f} k}{\rho c \eta_{f}},
\end{gathered}
$$

where $\sigma$ is the confining stress, $\eta_{H}$ and $\eta_{T}$ (both in $\mathrm{m}^{2} \mathrm{~s}^{-1}$ ) represent the hydraulic and thermal diffusivity, respectively, $\beta_{T}\left(\right.$ in $\mathrm{m}^{2} \mathrm{~Pa}^{-1} \mathrm{~s}^{-1}$ ) is the convective thermal diffusivity, the coefficient $1 / R$ represents a measure of the change in water content for a given change in pore fluid pressure when the porous material is permitted to drain freely, and $1 / H$ represents a measure of the change in water content for a given change in confining stress when the material is permitted to drain freely (Appendix A). The thermal expansivity coefficient $\alpha_{m}$ (in ${ }^{\circ} \mathrm{C}^{-1}$ ) describes how much fluid mass of water is expelled out of the porous material when the temperature is increased by $\left(T-T_{0}\right), \rho$ and $c$ are the bulk density (in $\mathrm{kg} \mathrm{m}^{-3}$ ) and the specific heat (in $\mathrm{J} \mathrm{m}^{-3}$ ${ }^{\circ} \mathrm{C}^{-1}$ ) of the porous medium, respectively, $\lambda$ is the thermal conductivity of the porous medium (in $\mathrm{W} \mathrm{m}^{-1}{ }^{\circ} \mathrm{C}^{-1}$ ), $\rho_{f}, c_{f}$, and $\eta_{f}$ represent the density (in $\mathrm{kg} \mathrm{m}^{-3}$ ), the specific heat (in $\mathrm{J} \mathrm{m}^{-3}{ }^{\circ} \mathrm{C}^{-1}$ ), and dynamic viscosity (in Pa s) of the pore fluid, respectively, and $k$ (in $\mathrm{m}^{2}$ ) is the permeability of the porous rock.

[16] We consider a buried thermomechanical source located at depth $z=b$. The boundary and initial conditions are $T=T_{0}+\delta T$ and $p=p_{0}+\delta p$ at $z=b$ and $T=T_{0}$, and $p=$ $p_{0}$ for $0 \leq z<b$, where $T_{0}$ and $p_{0}$ represent the temperature and pore fluid pressure distributions in the reference state and $\delta T$ and $\delta p$ represent a thermal disturbance and its associated pore fluid pressure variation. If the hydraulic diffusivity is high enough, all the excess fluid pressure generated by the increase of temperature dissipates as pore water flow. From equation (6) and the Darcy equation, the fluid pressure follows a classical diffusion-type equation [e.g., Palciauskas and Domenico, 1989]. For a 1-D model, this yields

$$
\frac{\partial^{2} p}{\partial z^{2}}=\frac{1}{\eta_{H}} \frac{d p}{d t}+\text { source terms }
$$

We consider that the initial distribution of fluid pressure in excess of hydrostatic (fluid overpressure) pressure follows $p(z, 0)-p_{0}=\delta p_{0} H(z-b)$, where $H(z)$ represents the unit step function $(H(z)=0$ for $z<0$ and $H(z)=1$ for $z \geq 0)$ and $\delta p_{0}$ represents an initial source pressure term. This source term can be either positive (e.g., an overpressure generated by compaction) or negative like in the case of fracturation related to dilatancy phenomena. Let us assume $\delta p_{0}>0$, the solution is:

$$
\delta p(z, t)=\frac{\delta p_{0}}{2 \sqrt{\pi \eta_{H} t}} \int_{b-z}^{\infty} \exp \left(-\frac{z^{\prime 2}}{4 \eta_{H} t}\right) d z^{\prime}
$$

where $\delta p(z, t)=p(z, t)-p_{0}$. This solution corresponds to a diffusive pressure front moving upward. The diffusive nature of the pressure front implies that it moves with a nonconstant velocity decreasing with the distance from the source. It should be noted that the velocity of displacement of this pressure front is independent of the strength of the source. This will not be the case for the shock waves analyzed below in sections 3.2 and 3.3.

\subsection{T-P Shock Waves (Type II)}

[17] If the hydraulic diffusivity of the porous material is small compared to the term $R \alpha_{m}$, equation (6) can be simplified as $\partial p / \partial t \approx R \alpha_{m}(\partial T / \partial t)$. This yields $p-p_{0}=$ $\left(R \alpha_{m}\right)\left(T-T_{0}\right)$, or $\delta p=\left(R \alpha_{m}\right) \delta T$. Therefore the pore fluid pressure change is linearly related to the temperature disturbance. To constrain how the pore fluid pressure change can, in turn, affect the material properties, let us examine the condition for hydrofracturation further. Assuming that the confining pressure is equal to the lithostatic pressure, the breakdown fluid overpressure is given by $\delta p_{F}=\gamma\left(P-p_{H}\right)+$ $T_{S}$, where $P$ is the lithostatic pressure, $p$ is the initial pore fluid pressure in the rock volume, $\gamma$ is the fracture coefficient for the rock ( 1.04 [e.g., Natale et al., 1998]), and $T_{S}$ is the tensile strength of the rock. In poorly compacted volcanic ashes $T_{S} \sim 0$ while jointed basaltic lava flows have in situ tensile strengths in the range $0.2-6 \mathrm{MPa}$ with the most common values $\sim 2-3 \mathrm{MPa}$ [Haimson and Rummel, 1982; Schultz, 1995]. At $2 \mathrm{~km}$ depth, taking $p=20 \mathrm{MPa}$, $T_{S}=10 \mathrm{MPa}$ as an upper bound, $\gamma=1$, and $P=40 \mathrm{MPa}$ yields $\delta p_{F} \approx 30 \mathrm{MPa}$. A sharp temperature increase $>100^{\circ} \mathrm{C}$ above the background temperature $T_{0}$ is responsible for a very high pore fluid pressure change $\delta p$, which can easily amount tens of megapascals (Appendix A). It follows that this pressure increase yields easily to fracturation of the rock directly in contact with the heat source. 
[18] For a 1-D model, inserting the linear temperature/ fluid pressure relationship $\partial p / \partial t \approx R \alpha_{m}(\partial T / \partial t)$ into equation (7) yields:

$$
\frac{\partial T}{\partial t}-\eta_{T} \frac{\partial^{2} T}{\partial z^{2}}-\beta_{T} R \alpha_{m}\left(\frac{\partial T}{\partial z}\right)^{2}=0
$$

Deriving this equation by $z$ and introducing the change of variable $c(z, t)=-2 \beta_{T} R \alpha_{m}(\partial T / \partial z)$ yields a Bürgers-type equation:

$$
\frac{\partial c}{\partial t}+c \frac{\partial c}{\partial z}=\eta_{T} \frac{\partial^{2} c}{\partial z^{2}}
$$

and

$$
c(z, 0)=c_{0} H(z-b) .
$$

Equation (15) represents an additive initial condition with $c_{0}=c(z, 0)=-2 \beta_{T} R \alpha_{m}(\partial T / \partial z)_{0}$ representing the initial thermal shock and $b$ is the depth of the heat source (Figure 2). Note that $c$, like $\mathbf{u}$, has the dimension of a velocity (in $\mathrm{m} \mathrm{s}^{-1}$ ). A solution of the previous Bürgers's problem [e.g., Garcia and Natale, 1999] is,

$$
c(z, t)=\frac{c_{0}}{2}\left\{1-\tanh \left[\frac{c_{0}}{4 \eta_{T}}\left(z-\frac{c_{0} t}{2}\right)\right]\right\} .
$$

This solution corresponds to the propagation of a thermomechanical shock wave traveling upward at constant velocity and amplitude, both of them being interdependent. Equation (16) has the classical form of a wave $c(z, t)=\mathrm{f}(z-$ $V t)$ with a velocity given by $V=c_{0} / 2=-\beta_{T} R \alpha_{m}(\partial T / \partial z)_{0}$ and $c_{0}$ corresponds, in addition, to the wave amplitude. Higher is the initial velocity (i.e., higher is the initial thermal shock), higher is the wave velocity. This characteristic is very different from that discussed in section 3.1 for Type I disturbances. An order of magnitude of the wave velocity is few hundreds of meters per year. Such a shock wave carries up a fluid pressure gradient, which is maintained by the associated rock-pore water temperature gradient. However, a complete analysis of this phenomenon would imply, in principle, the use of a micromodel in which the generation and propagations of cracks are properly modeled in the computation of the fluid pressure. For example, the effect of pore fluid pressure and fracturation should be explicitly included into the variation of $\beta_{T}$ (through the dependence of the permeability) and $R$. A physical model of hydrofracturation has been described very recently [Flekkøy et al., 2002], which could be used for this purpose.

\subsection{Pressure Shock Waves (Type III)}

[19] A volcanic system can contain several aquifers with impermeable boundaries due to self-organized dissolution/ precipitation reactions in the hydrothermal system. In this case, a volcanic system can be assimilated to a compartmentalized system like those observed in sedimentary basins. We consider a sudden increase of the pore fluid pressure inside an aquifer due to temperature or confining stress disturbances associated with the presence of a magmatic intrusion in its vicinity. In undrained conditions, fluid pressure increases in

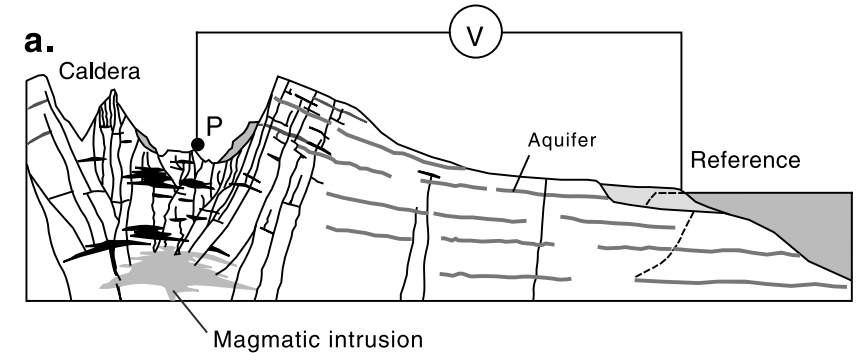

b.

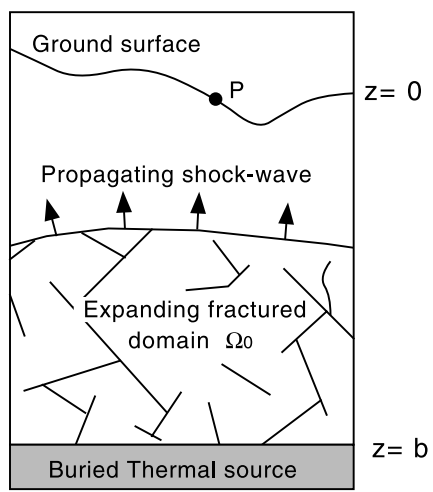

C.

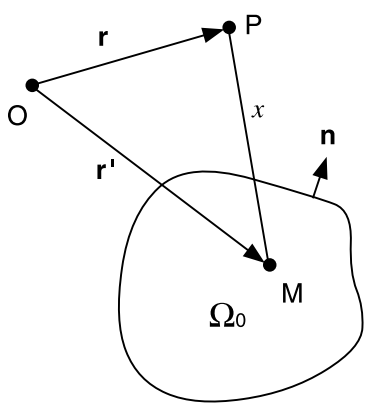

Figure 2. THM disturbances in a volcanic system. (a) The self-potential corresponds to electrical potential scanned at the ground surface using nonpolarizable electrodes. There is the need for a reference station located as far as possible from the perturbed area or the installation of dipoles of measurements to look at the electrical field. (b) Propagation of a shock wave from a THM source located at depth $b$ toward the ground surface. (c) Sketch of the source volume $\Omega_{0}$ containing the electrokinetic sources ( $M$ corresponds to the position of the current source point) and the observation station $P$ located at the ground surface.

the aquifer until the condition for fracturation is reached. We assume a first-order Taylor development of the variation of the hydraulic diffusivity with the pore fluid pressure $\eta_{H}=$ $\eta_{0}\left(1+h\left(p-p_{0}\right)+\ldots\right)$, where $h>0$ represents a fracturing parameter. For $p \sim p_{0}$ at the head of the wave, equation (6) leads to [e.g., Revil, 2002b]:

$$
\frac{\partial p}{\partial t}-\eta_{0} \frac{\partial^{2} p}{\partial z^{2}}-\eta_{0} h\left(\frac{\partial p}{\partial z}\right)^{2}=0
$$

Deriving equation (17) by $z$ yields again a Bürgers equation:

$$
\frac{\partial c}{\partial t}+c \frac{\partial c}{\partial z}=\eta_{0} \frac{\partial^{2} c}{\partial z^{2}}
$$

and

$$
c(z, t)=-2 \eta_{0} h \frac{\partial p}{\partial z}
$$

The initial condition can be written as $c(z, 0)=c_{0} H(z-b)$ for $z>0$ and $c_{0}$ is given by:

$$
c_{0} \equiv-2 \eta_{0} h\left(\frac{\partial p}{\partial z}\right)_{0}
$$


(the fluid pressure gradient source follows the step function). Solving equation (18) with initial condition (20) yields:

$$
c(z, t)=\frac{c_{0}}{2}\left\{1-\tanh \left[\frac{c_{0}}{4 \eta_{H}}\left(z-\frac{c_{0} t}{2}\right)\right]\right\} .
$$

This solution corresponds again to the propagation of a hydromechanical shock wave traveling upward at constant velocity $V=c_{0} / 2$ and amplitude $c_{0}$. An order of magnitude is obtained with $\eta_{f}=10^{-4} \mathrm{~Pa} \mathrm{~s}, k_{0}=10^{-15} \mathrm{~m}^{2}(1 \mathrm{mD}), R=$ $1000 \mathrm{MPa},(\partial p / \partial z)_{0}=1 \mathrm{MPa} \mathrm{m}{ }^{-1}, h=10^{-3} \mathrm{~Pa}^{-1}$. This yields $V=10 \mathrm{~m} \mathrm{~s}^{-1}$. This is of very high speed indicating that shock waves of Type III move up more efficiently in the volcanic system than shock waves of Type II.

\section{Electrical Disturbances}

[20] The THM disturbances described in section 3 create variations of electrical current in the system owing to the electrokinetic coupling. Simple computations of the order of magnitude show that the disturbances of Type II are associated with a source current density equal to $0.1 \mathrm{~mA}$ $\mathrm{m}^{-2}$ and disturbances of Type III with a source current density of $1 \mathrm{~A} \mathrm{~m}^{-2}$ at the forefront of the shock wave. This implies that significant electromagnetic disturbances are associated with the development of these THM disturbances. Therefore now the basic idea is to relate the Maxwell equations to the THM disturbances via the electrokinetic coupling.

\subsection{Electromagnetic Disturbances}

[21] The Maxwell equations in a homogeneous piece of porous material at rest (i.e., the velocity of the matrix framework is small by comparison with the speed of light) are:

$$
\begin{gathered}
\varepsilon \nabla \cdot \mathbf{E}=\rho, \\
\nabla \times \mathbf{E}+\mu \frac{\partial \mathbf{H}}{\partial t}=0, \\
\nabla \times \mathbf{H}-\varepsilon \frac{\partial \mathbf{E}}{\partial t}=\mathbf{j}, \\
\nabla \cdot \mathbf{H}=0,
\end{gathered}
$$

where $\rho$ is the net charge density, $\varepsilon$ is the dielectric constant of the porous material, $\mu$ is the magnetic permeability, $\mathbf{H}$ is the magnetic field, and $\mathbf{j}=\sigma \mathbf{E}+\mathbf{j}_{S}$ is the total current density (Ohm's law plus electrokinetic source term). This yields:

$$
\begin{gathered}
-\nabla \times \nabla \times \mathbf{E}-\frac{1}{\eta} \frac{\partial \mathbf{E}}{\partial t}-\mu \frac{\partial \mathbf{j}_{S}}{\partial t}=\frac{1}{c^{2}} \frac{\partial^{2} \mathbf{E}}{\partial t^{2}}, \\
-\nabla \times \nabla \times \mathbf{H}-\frac{1}{\eta} \frac{\partial \mathbf{H}}{\partial t}+\nabla \times \mathbf{j}_{S}=\frac{1}{c^{2}} \frac{\partial^{2} \mathbf{H}}{\partial t^{2}},
\end{gathered}
$$

where $\eta \equiv 1 /(\sigma \mu)$ is the diffusivity of the electromagnetic surges in the low-frequency limit and $c^{2} \equiv 1 /(\varepsilon \mu), c$ is the velocity of the electromagnetic disturbances in the high-frequency limit ( $c$ is a complex, $\varepsilon=\varepsilon^{\prime}-i \varepsilon^{\prime \prime}, \omega$ is the angular frequency, and $i^{2}=-1$ ). Equations (26) and (27) contain terms which are characteristic of diffusive and wave equations. A critical frequency $f_{c}$ can be defined between these two domains by $f_{c}=\omega_{c} / 2 \pi$, where $\omega_{c}=\sigma / \varepsilon^{\prime}$.

[22] Now we look at the frequency dependence of the excitation source term. We consider that the fluid pressure obeys harmonic fluctuations, i.e., $p(r, t)=p(r) \exp (-i \omega t)$. Then the flow rate and the source current density are also oscillatory functions:

$$
\begin{gathered}
\mathbf{u}=-\frac{k(\omega)}{\eta_{f}}\left(\nabla p(\mathbf{r}) \exp (-i \omega t)-\rho_{f} \mathbf{g}\right), \\
\mathbf{j}_{S}=-L(\omega) \nabla p(\mathbf{r}) \exp (-i \omega t),
\end{gathered}
$$

where $k(\omega)$ and $L(\omega)$ are the dynamic permeability and electrokinetic coupling terms, respectively. These parameters are complex-valued and frequency dependent in the frequency region where there is a competition between viscous effects and inertial effects in the porous network, that is when the viscous skin depth $\delta=\left(2 \eta_{f} / \rho_{f} \omega\right)^{1 / 2}$ is comparable to a characteristic radius $\Lambda$ of the porous network [Kostek et al., 1992]. Pride [1994] determined the frequency dependence of both $k$ and $L$ as:

$$
\begin{gathered}
k(\omega)=k_{0}\left[\left(1-i \frac{\omega}{\omega_{c}} \frac{4}{M}\right)^{1 / 2}-i \frac{\omega}{\omega_{c}}\right]^{-1}, \\
L(\omega) \approx L_{0}\left(1-i \frac{\omega}{\omega_{c}} \frac{M}{4}\right)^{-1 / 2},
\end{gathered}
$$

where $M$ is a pore geometry-dependent dimensionless parameter, $2 \leq M \leq 8$ for a wide class of porous materials ranging from granular media to networks of connected capillaries [e.g., Kostek et al., 1992] and $\omega_{c}$ is here a new critical frequency separating low-frequency viscous flow and high-frequency inertial flow:

$$
\omega_{c} \equiv \frac{\eta_{f}}{F \rho_{f} k_{0}} .
$$

In equation (32) $F$ represents the electrical formation factor defined as the ratio between the tortuosity of the pore space and the porosity. An order of magnitude of $\omega_{c}$ is obtained using $F=10, k_{0}=10^{-12} \mathrm{~m}^{2}(1 \mathrm{mD}), \rho_{f}=1000 \mathrm{~kg} \mathrm{~m}^{-3}$, and $\eta_{f}=10^{-3} \mathrm{~Pa} \mathrm{~s}$. This yields $f_{c}=\omega_{c} / 2 \pi \sim 10 \mathrm{kHz}$. In the high frequency domain $\left(\omega \gg \omega_{c}\right)$, the source current density depends on the frequency as $L(\omega) \exp (-i \omega t)$. Therefore above the critical frequency $\omega_{c}$, the source current density decreases with the frequency. In other words, any groundwater flow fluctuations cannot generate electromagnetic signals with a frequency contend much above the critical frequency $\omega_{c} / 2 \pi$. We conclude that it is safe to consider the 
Maxwell equations only in their low-frequency diffusive modes.

\subsection{Low-Frequency Electrical Signals}

[23] In the low-frequency domain $\left(f \ll f_{c} \sim 10^{10} \mathrm{~Hz}\right)$, the electrical field is solution of:

$$
-\nabla \times \nabla \times \mathbf{E}-\frac{1}{\eta} \frac{\partial \mathbf{E}}{\partial t}-\mu \frac{\partial \mathbf{j}_{S}}{\partial t}=0 .
$$

Using the Lorentz gauge, the electrical and magnetic fields are given by:

$$
\begin{gathered}
\mathbf{E}=-\nabla \varphi-\frac{\partial \mathbf{A}}{\partial t}, \\
\mathbf{B}=\mu \mathbf{H}=\nabla \times \mathbf{A}, \\
\nabla \cdot \mathbf{A}+\frac{1}{c^{2}} \frac{\partial \varphi}{\partial t}=0,
\end{gathered}
$$

and $\mathbf{A}(\infty)$ and $\varphi(\infty)$ represent the magnetic vector and the electrical potential far from the source volume. Equations (33)-(36) yield:

$$
\nabla^{2} \varphi-\mu \varepsilon \frac{\partial^{2} \varphi}{\partial t^{2}}=\frac{1}{\sigma} \nabla \cdot \mathbf{j}_{S}+\frac{1}{\sigma} \frac{\partial \rho}{\partial t}
$$

We note $\tau$ as the time for an electromagnetic disturbance to diffuse between two points separated by a characteristic distance $L$. We have $\tau=L^{2} / \eta=L^{2} \sigma \mu$. Taking $L=1 \mathrm{~km}$, $\sigma=0.1 \mathrm{~S} \mathrm{~m}^{-1}$, and $\mu \sim 10^{-6} \mathrm{H} \mathrm{m}^{-1}$, we obtain $\tau=0.1 \mathrm{~s}$. Consequently, the electrical disturbances diffuse with a very high velocity in the conductive volcanic rock. At the ground surface, the record of these electrical field variations (one properly filtered to remove extraneous contributions like those associated with telluric currents, for example) brings quasi-instantaneous information about the location of the THM disturbances. Therefore an analysis of the distribution of this electrical field at the ground surface could be used to determine the location of the hydraulic source using dedicated tomographic algorithms as shown in section 6 . In the quasi-static limit, we obtain:

$$
\nabla^{2} \varphi=\frac{1}{\sigma} \nabla \cdot \mathbf{j}_{S}
$$

[24] Now we analyze the electrical potential associated with the propagation of a fracturation front in the volcanic system. We consider the system formed by a piecewise uniform conducting half-space comprising two regions $\Omega_{0}$ and $\Omega_{1}$ with constant electrical conductivities $\sigma_{0}$ and $\sigma_{1}$. The region $\Omega_{0}$ confined by the surface $\partial \Omega$, represents the expanding fractured domain. The source volume $\Omega_{0}$ contains a source of current $\mathbf{j}_{S}$ and the total electrical current density is given by:

$$
\mathbf{j}=\sigma_{0} \mathbf{E}+\mathbf{j}_{S}
$$

and

$$
\mathbf{j}_{\mathrm{S}} \equiv \frac{\eta_{f} L}{k} \mathbf{u}
$$

The continuity equation $\nabla \cdot \mathbf{j}=0$ written in the quasi-static limit combined with equation (39) yields $\nabla \cdot\left(\sigma_{0} \mathbf{E}\right)=-\nabla$. $\mathbf{j}_{s}$. Therefore the electrical potential $\varphi$ obeys:

$$
\begin{gathered}
\nabla^{2} \varphi=\frac{\left(\nabla \cdot \mathbf{j}_{S}\right)}{\sigma_{0}}, \text { in } \Omega_{0}, \\
\nabla^{2} \varphi=0, \text { in } \Omega_{1}, \\
\sigma_{1} \mathbf{n} \cdot \nabla \varphi_{1}-\sigma_{0} \mathbf{n} \cdot \nabla \varphi_{0}=\mathbf{j}_{S} \cdot \mathbf{n}, \text { on } \partial \Omega, \\
\varphi_{1}=\varphi_{0}, \text { along } \partial \Omega .
\end{gathered}
$$

The electrokinetic coupling coefficient $C$ is defined by:

$$
C \equiv\left(\frac{\partial \varphi}{\partial p}\right)_{\mathbf{j}=0}=-L / \sigma .
$$

[25] The coefficient $C$ represents the sensitivity coefficient of electrical potential with pore fluid pressure. Its magnitude is investigated in section 5 . We note as $\delta p$ the pore fluid pressure in excess of the reference fluid pressure defined in the reference state. Now the question is to know if there is a drop in the streaming potential coupling coefficient at the interface $\partial \Omega$ between the fractured and undisturbed domains. We use the model of Revil [2002c] that includes a percolation threshold in the rock material properties. This yields:

$$
\begin{gathered}
L_{0} \approx-\frac{\varepsilon_{f} \zeta}{\eta_{f} F}, \\
k_{0}=\frac{d^{2}}{\alpha F(F-1)^{2}}, \\
F=\left(\phi-\phi_{p}\right)^{-3 / 2},
\end{gathered}
$$

where $\varepsilon_{f}$ is the dielectric constant of the pore water (in $\mathrm{F}$ $\left.\mathrm{m}^{-1}\right), F$ is the formation factor entering the electrical conductivity problem, $\zeta$ is the zeta potential, an electrochemical property of the mineral/pore water interface [Revil and Leroy, 2001], $d$ is a mean grain or fragment size, $\alpha$ is a numerical constant $(\alpha \sim 8), \phi$ represents the porosity, and $\phi_{p}$ the percolation porosity (i.e., the porosity at the percolation threshold corresponding to the loss of interconnectivity of the porous network). If we consider that in the undisturbed volume, we are close to a percolation threshold (i.e., $\phi \sim \phi_{p}$ ), both $k$ and $L$ are very small in region $\Omega_{1}$. Therefore the propagation of a fracturation front 
(Types II and III) increases dramatically both $k$ and $L$. It follows that there is a drop in the streaming potential coupling coefficient at the expanding fracturation front $\partial \Omega$. This is not the case for the diffusion of a pressure front (Type I) without fracturation of the rock. The only exception corresponds to the inflow of water in a dilatant (fractured) domain.

[26] The scalar function $C \cdot \delta p$ is a twice-differentiable function of position within $\Omega_{0}$, zero outside $\Omega_{0}$. The continuity equation $\nabla \cdot \mathbf{j}=0$ yields $\nabla \cdot\left(\sigma_{0} \mathbf{E}+\mathbf{j}_{S}\right)$, and it follows that $\nabla^{2} \psi=0$, in $\Omega_{0}$, where $\psi=\varphi+C \cdot \delta p$ is an effective potential [Fitterman, 1978]. It follows that $\psi$ is harmonic inside and outside $\Omega_{0}$, and it obeys the following boundary value problem:

$$
\begin{gathered}
\nabla^{2} \psi=0, \text { in } \Omega_{0}, \text { and } \Omega_{1}, \\
\psi_{1}-\psi_{0}=-C \cdot \delta p \text {, on } \partial \Omega, \\
\sigma_{1} \mathbf{n} \cdot \nabla \psi_{1}=\sigma_{0} \mathbf{n} \cdot \nabla \psi_{0}, \text { along } \partial \Omega .
\end{gathered}
$$

Therefore the volume distribution of current in the source region $\Omega_{0}$ is equivalent to a dipole layer lying on the boundary of the source body $\delta \Omega$ (Figure 3 ). The potential drop $\psi_{1}-\psi_{0}=-C \cdot \delta p$ corresponds to the dipolar momentum of the source. Note that outside the source volume, $\psi=\varphi$. It follows that a shock wave is responsible for an electrical field in the whole space surrounding the disturbance. The strength of the source $-C \cdot \delta p$ is controlled both by the coupling coefficient $C$ and the strength of the pressure pulse. As $|C|>0.1 \mathrm{~V} \mathrm{MPa}^{-1}$ (see section 5) a positive pressure pulse of say $20 \mathrm{MPa}$ generates a source term $\Delta \varphi>2 \mathrm{~V}$. The electrical potential at the observation station $P$ located outside the source volume (usually $P$ will be located at the ground surface) is given from equations (49)-(51) as:

$$
\varphi(P, t)=-\frac{C}{2 \pi} \int_{\partial \Omega} \delta p\left(r^{\prime}, t\right)\left(\frac{\mathbf{x} \cdot \mathbf{n}}{x^{3}}\right) d S-\frac{1}{2 \pi} \int_{\Omega} \frac{\mathbf{E}\left(\mathbf{r}^{\prime}, t\right)}{x} \cdot \frac{\nabla \sigma}{\sigma} d V
$$

where $\mathbf{x} \equiv \mathbf{r}-\mathbf{r}^{\prime}$ and $x=|\mathbf{x}|$ represents the distance between the observation point $P$ and the infinitesimal volume element $d V$ of the medium surrounding the integration point $M \in \Omega_{0}, \mathbf{n}$ is the outward normal to the interface $\partial \Omega$ at the current source point $M$ shown in Figure $3, d S$ is a surface element of the interface on $\partial \Omega$, around $M$ (Figure 3 ), $\delta p\left(\mathbf{r}^{\prime}, t\right)$ is the excess pore fluid pressure at the source point $M$. The first term of equation (52) corresponds to the primary source term associated with the propagation of the pressure front whereas the second term corresponds to secondary sources associated with accumulation of charge at electrical conductivity heterogeneities. Of course, this electrical field disturbance is to add to the electrical field contribution observed in the reference state discussed in section 2 (see equation (5)). The primary source term varies as $1 / x^{2}$ from the source, whose dependence is typical of a dipolar source.
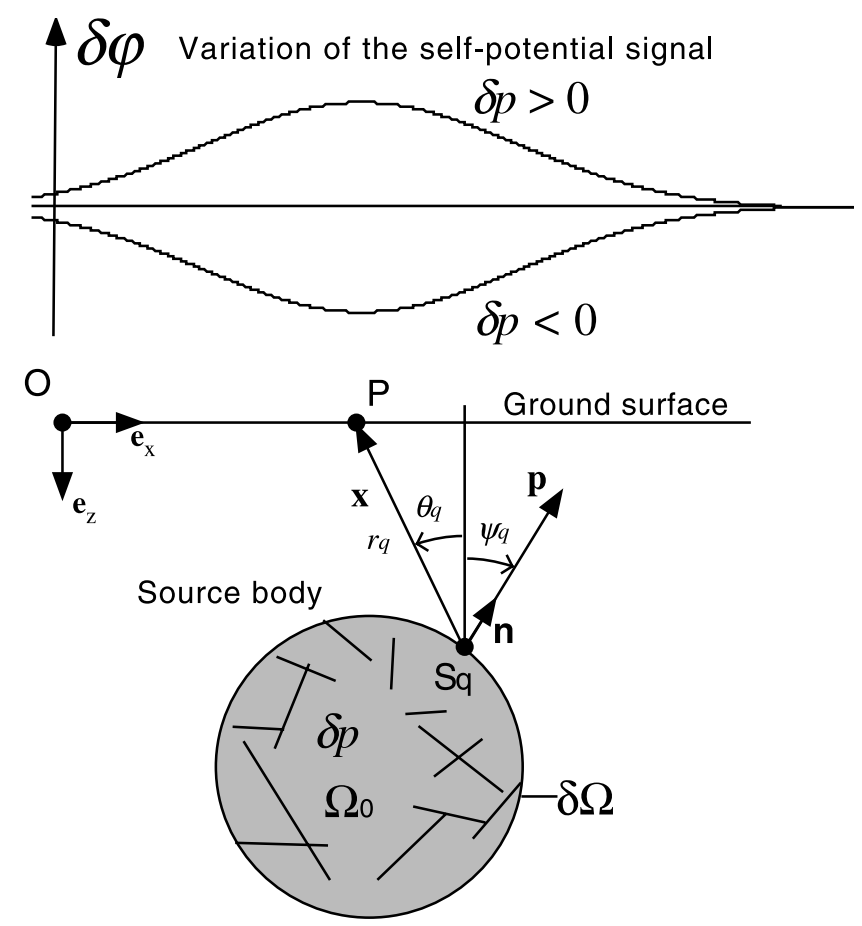

Figure 3. Sketch of the variation of the self-potential signal recorded at the ground surface and associated with the extension of a fracturation front at depth. We noted $\mathbf{p}$, the dipolar momentum, $S_{q}$, a source point located on the surface of the source body, and $P$, an observation station located at the ground surface. For electrokinetic sources, the dipolar momentum is usually in the direction of groundwater flow, which depends in turn on the sign of the pressure source $\delta p$. If $C$ is negative, a positive pressure source $\delta p>0$ generates a positive self-potential signal variation $\delta \varphi$ at the ground surface.

[27] An alternative to the measurements of the selfpotential concerns the measurements of the electrical field, which is everywhere tangent along the ground surface (the normal component vanishes due to the fact that the atmosphere is insulating). The electrical field is given by:

$$
E_{u}(P, t) \equiv-\frac{\partial \varphi(P, t)}{\partial u}=-\left[\frac{\partial \varphi}{\partial x}+\frac{\partial \varphi}{\partial z} \frac{d z}{d x}\right] \frac{d x}{d u}
$$

where $u$ is the curvilinear coordinate, $d z / d x$ is the slope effect of the curvilinear elevation profile describing the ground surface, and $\varphi(P, t)$ is given by equation (52). The advantage of electrical field measurements is that it does not require the installation of long wires. Indeed, the electrical field could be measured locally using two sets of electrodes perpendicularly disposed and using a network of such measurements dipoles independent of each other. The length of each dipole should be optimized to get the best signal-to-noise ratio.

\section{Magnitude of the Coupling Coefficient}

[28] Here we investigate the order of magnitude of the streaming potential coupling coefficient $C$ of different materials of interest in volcanic context. The dependence 
of $C$ with $\mathrm{pH}$ and salinity for fractured basaltic rocks, scoria, and siliciclastic zeolitized materials is important as both the salinity and the $\mathrm{pH}$ can vary over a wide spectrum in active volcanic areas. The data are interpreted with the model developed by Revil et al. [2002b]. The samples are crushed basalts (primarily composed of plagioclase, feldspar, and pyroxene), scoria (from Stromboli), and two crushed tuff samples. Sample Bu-96-8 is a clay-free zeolite-rich sample, whereas Bu-96-7 is a smectite-rich zeolite-rich sample (sample Bu-96-7: 61.0\% clinoptilolite, 11\% smectite, 18.5\% feldspar, 7.6\% quartz; sample Bu-96-8: 62.0\% clinoptilolite, $8.5 \%$ feldspar, $14.9 \%$ quartz, and $9.4 \%$ plagioclase, determined from semiquantitative XRD analysis [see Revil et al., 2002b]).

[29] We use a $\mathrm{NaCl}$ solution for the background electrolyte. The $\mathrm{pH}$ is controlled by adding $\mathrm{NaOH}$ or $\mathrm{HCl}$ to the electrolyte. The $\mathrm{pH}$ and salinity are monitored prior and after each streaming potential and electrical conductivity measurements. All the experiments are done at room temperature, $\sim 21^{\circ} \mathrm{C}$. Each sample is immersed in a solution of the desired salinity and $\mathrm{pH}$ during $40-80$ hours prior to each experiment to be sure that thermodynamic equilibrium is reached. We believe that many experiments reported in the literature in which the pore water solutions are changed too abruptly do not reflect equilibrium situations and should be considered with caution. The experimental setup used for the electrokinetic experiments is shown in Figure 4a. The streaming potential coupling coefficient is determined from the slope of the electrical potential versus the pore fluid pressure drop between the two reservoirs (Figures $4 b$ and $4 c$ ).

[30] The experimental results are displayed in Figures $4 d$ to $4 \mathrm{f}$ and Figures $5 \mathrm{a}$ to $5 \mathrm{~d}$ (each point represents a coupling coefficient value defined from a set of measurements as shown in Figure 4c). Results are relatively similar to that obtained by Lorne et al. [1999] for silica-dominated rocks. We found that $C$ is inversely proportional to the pore water conductivity (or salinity) over several orders of magnitude. At $\mathrm{pH}>3.4$, the coupling coefficient $C$ of basalt is negative, which means that the flow of groundwater carries a dipolar moment pointing in the flow direction. Below $\mathrm{pH} \mathrm{3.4,C}$ becomes positive for basalts and the dipole moment points in the direction opposite to the groundwater flow. The strength of the coupling coefficient $C$ is the highest at low brine salinity and near neutral $\mathrm{pH}$ conditions. However, even at high salinity, the strength of $C$ remains high. For example $C \sim-100 \mathrm{mV} \mathrm{MPa}^{-1}$ at a salinity of $0.01 \mathrm{~mol}^{-1}$. This is a high value and this means that a fluid pressure pulse of $20 \mathrm{MPa}$ can generate an electrical source pulse $\sim 2 \mathrm{~V}$.

[31] The streaming potential coupling coefficients $C$ versus salinity and $\mathrm{pH}$ of the tuff samples are reported in Figures $5 \mathrm{a}$ and $5 \mathrm{~b}$. We observe that $C$ is inversely proportional to the logarithm of the salinity. In addition to the electrokinetic experiments, we also performed electrical conductivity experiments at a frequency of $1 \mathrm{kHz}$ using the same procedure and apparatus as described by Revil et al. [2002b] to determine both the electrical formation factor and surface conductivity. The validity of using crushed samples to determine both zeta potential and surface conductivity can be assessed by comparing measurements made on intact and crushed samples. For sample Bu-96-8, zeta potential and surface conductivity for the crushed sample are $-17 \mathrm{mV}$ and $0.4 \times 10^{-3} \mathrm{~S} \mathrm{~m}^{-1}$ and $-16 \mathrm{mV}$ and $2.9 \times$
$10^{-3} \mathrm{~S} \mathrm{~m}^{-1}$ for the intact sample (porosity 0.155 ) [Revil et al., 2002b]. For sample Bu-96-7, zeta potential and surface conductivity for the crushed sample are $-32 \mathrm{mV}$ and $57 \times$ $10^{-3} \mathrm{~S} \mathrm{~m}^{-1}$, respectively, and $-33 \mathrm{mV}$ and $90 \times 10^{-3} \mathrm{~S}$ $\mathrm{m}^{-1}$, respectively, for the intact sample (porosity 0.30 ). So once properly corrected for surface conductivity, the value of the zeta potential is the same for intact and crushed samples. This means that the zeta potential can de determined on crushed sample and then used with electrical conductivity data performed on the intact sample to determine the streaming potential coupling coefficient of the intact sample. This appears a powerful way to proceed, as streaming potential measurements are much simpler to carry out with crushed samples. This is of course true as long as the crushed sample is representative of the intact sample for the minerals exposed to the flow of the pore water.

[32] We are now looking for the influence of a temperature change $\delta T$ upon electrical signals in a thermoporoelastic water-saturated medium. To define the magnitude of the efficiency of the electrokinetic conversion, we compute the effect of a fast temperature increase in a water-saturated rock volume upon the intensity of electrical potential generated in drained conditions. For this purpose, we introduce a sensitivity coefficient $C_{T}$ between the electrical potential and the temperature:

$$
C_{T} \equiv\left(\frac{\partial \varphi}{\partial T}\right)_{\mathbf{j}=0}=\left(\frac{\partial \varphi}{\partial p} \frac{\partial p}{\partial T}\right)_{\mathbf{j}=0}=C\left(\frac{\partial p}{\partial T}\right)_{\mathbf{j}=0},
$$

where $C_{T}$ represents the electrical potential increase through electrokinetic coupling per unit degree Celsius of temperature increase in a water-saturated porous material when the porous material is permitted to drain freely. According to equations (A21) and (A22), the coefficient $C_{T}$ can be evaluated with

$$
Q\left(\alpha_{b} \xi+\alpha_{m}\right) C \leq C_{T} \leq R \alpha_{m} C .
$$

For a basalt, we obtain $C_{T} \sim-10 \mathrm{mV}{ }^{\circ} \mathrm{C}^{-1}$ with a groundwater electrical conductivity $\sigma_{f}=1 \mathrm{~S} \mathrm{~m}^{-1}$ and $C_{T} \sim$ $-100 \mathrm{mV}^{\circ} \mathrm{C}^{-1}$ with a pore fluid conductivity $\sigma_{f}=0.1 \mathrm{~S} \mathrm{~m}^{-1}$. Therefore a temperature change of $100^{\circ} \mathrm{C}$ by comparison with the background temperature yields an electrical source term comprised between 1 and $10 \mathrm{~V}$. Therefore the THM coupling represents an extremely efficient way to generate transient self-potential signals in active volcanoes.

\section{Tomographic Algorithms}

[33] Now that the forward problem and sensitivity of the electrical signals have been evaluated, we focus on the tomographic problem. In the last years, new tomographic methods have been developed to locate buried sources of electrical potential fields [e.g., Sailhac and Marquis, 2001; Gibert and Pessel, 2001]. Our purpose is to define a simple imaging method to locate the depth of the source of polarization responsible for the electrical field disturbance measured at the ground surface.

[34] Patella [1997] proposed a tomographic algorithm to locate the causative buried sources responsible for the selfpotential signals recorded at the ground surface. It is an intercorrelation method used to define a density probability 

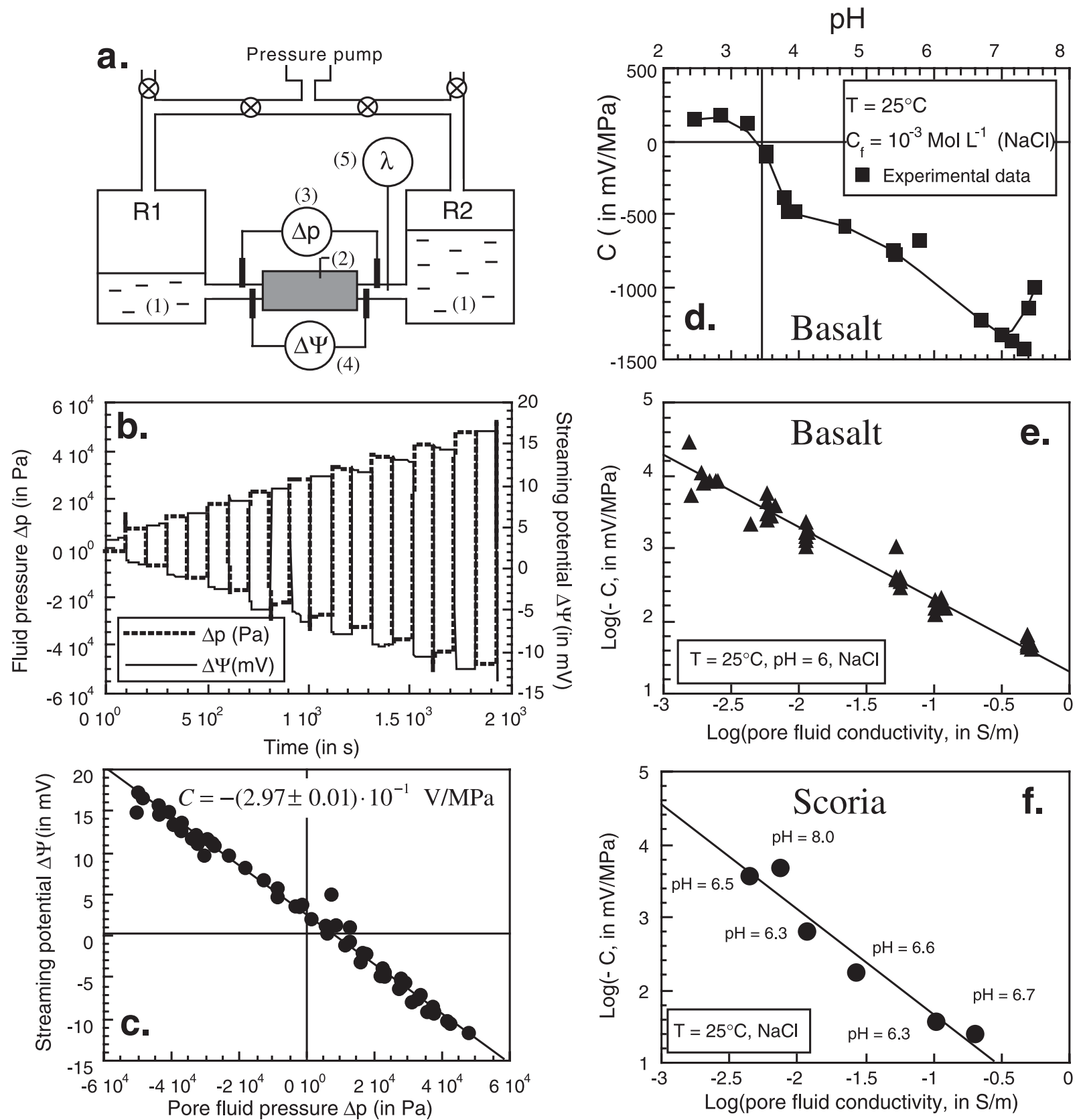

Figure 4. Laboratory measurements of the electrokinetic coupling coefficient. (a) Sketch of the experimental setup (ZetaCad ${ }^{(i)}$ ): (1) pore fluid reservoirs $R 1$ and $R 2$; (2) sample tube; (3) pressure sensors; (4) voltage nonpolarizable electrodes connected to an impedance meter; and (5) measurements of the electrical conductivity of the electrolyte. The pressure is controlled with nitrogen gas, which has no effect on the $\mathrm{pH}$ and salinity of the electrolyte. All the equipments are directly controlled by a desktop computer, and the experimental conditions and results are directly written in a file. (b) Example of a typical run. The system records the electrical potential drop generated at the end faces of a sample during electrolyte flow under a fixed fluid pressure gradient. Note that the pressure gradient can be negative as by convention $\Delta p$ represent the fluid pressure difference between the two labeled compartments $R 1$ and $R 2$. (c) The streaming potential coupling coefficient is given as the slope of the recorded electric potential versus the fluid pressure differentials imposed between the end faces of the sample. (d) Electrokinetic coupling coefficient of crushed basalts (porosity $\sim 0.40$ ) versus $\mathrm{pH}$ at constant salinity. (e) Coupling coefficient of crushed basalts versus brine conductivity at constant $\mathrm{pH}$. A linear regression yields $\log _{10}(-C)=1.12-1.08 \log _{10} \sigma_{f}$ where $C$ is in $\mathrm{mV} \mathrm{MPa}^{-1}$ and $\sigma_{f}$ in $\mathrm{S} \mathrm{m}^{-1}$. (f) Coupling coefficient of scoria versus brine conductivity. A linear regression yields $\log _{10}(-C)=0.23-1.44 \log _{10} \sigma_{f}$. 

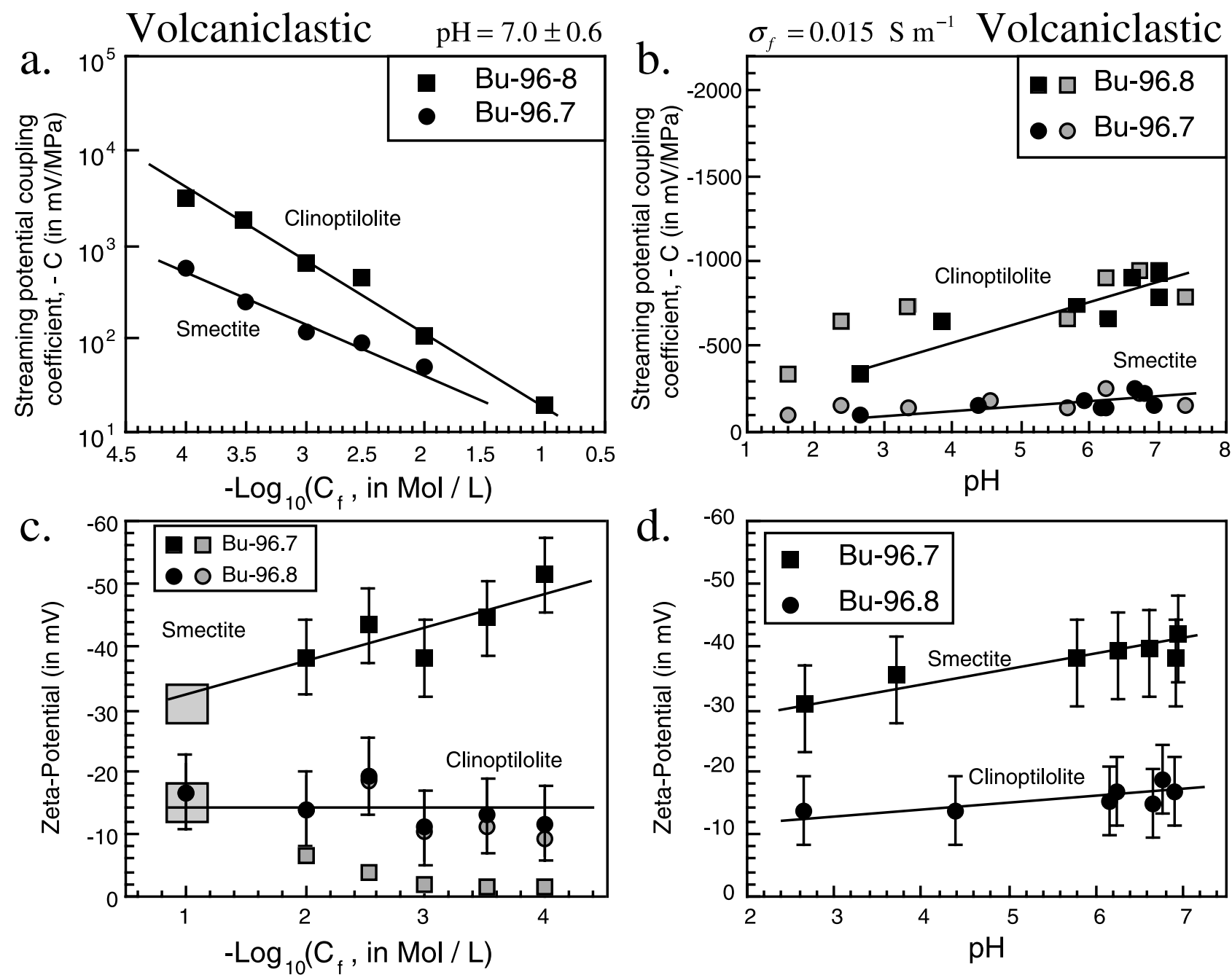

Figure 5. (a) Streaming potential coupling coefficient of volcaniclastic rocks versus salinity. The error bars are smaller than the size of the symbols. (b) Streaming potential coupling coefficient versus $\mathrm{pH}$. The error bars are smaller than the size of the symbols. The open and filled symbols correspond to the initial and final $\mathrm{pH}$, respectively. (c) Zeta potential versus salinity. The open symbols correspond to apparent zeta potential (i.e., not corrected for surface conductivity) whereas filled symbols correspond to surface corrected zeta potential values. The gray filled squares correspond to the measurements made by Revil et al. [2002b] with consolidated rock samples Bu-96-7 and Bu-96-8 at $C_{f}=3 \times 10^{-4} \mathrm{~mol} \mathrm{l}^{-1}$. (d) Zeta potential versus $\mathrm{pH}$ for the two volcaniclastic samples (corrected for surface conductivity).

of occurrence of the source at depth. One of the drawbacks of this method lies in the fact that Patella decomposed the source as a sum of monopoles. However, according to the statements made in sections 3-6, electrokinetic sources tend to produce locally dipolar separations of charges. Therefore here we consider the THM source as a sum of dipoles with strength proportional to $-\delta p C$. The electrical potential $\varphi$ (in V) at the observation station $P$ generated by the primary source term can be represented as a sum of $Q$ electrostatic dipoles in a homogeneous rock volume:

$$
\begin{gathered}
\varphi_{q}(P)=\sum_{q=1}^{Q} \frac{p_{q}}{4 \pi \varepsilon r_{q}^{2}}\left[\cos \left(\theta_{q}+\psi_{q}\right)\right], \\
\varphi_{q}(P)=\sum_{q=1}^{Q} \frac{p_{q}}{4 \pi \varepsilon r_{q}^{2}}\left[\cos \theta_{q} \cos \psi_{q}-\sin \theta_{q} \sin \psi_{q}\right],
\end{gathered}
$$

where $p_{q}$ is the dipolar momentum of source $q$ (in $\mathrm{C} \mathrm{m}$ ), $\varepsilon$ is the dielectric constant of the medium (in $\mathrm{F} \mathrm{m}^{-1}$ ), and $r_{q}$ is the distance (in $\mathrm{m}$ ) between the dipole $q$ and the observation point $P[x, z(x)], z(x)$ represents the topography of the ground surface, and $\left(x_{q}, z_{q}\right)$ are the coordinates of the $q$ th source, and $0_{q}$ and $\psi_{q}$ are two angles defined in Figure 3. The selfpotential measured along the local curvilinear coordinate $u$ at point $P$ located at the ground surface (outside the source volume containing the primary sources) can be written as the sum of $Q$ discretized electrical dipoles located in depth. This yields:

$$
\varphi(P)=\sum_{q=1}^{Q} \frac{-\Gamma_{q, 1}\left(x-x_{q}\right)-\Gamma_{q, 2}\left(z(x)-z_{q}\right)}{\left[\left(x-x_{q}\right)^{2}+\left(z(x)-z_{q}\right)^{2}\right]^{3 / 2}},
$$

where $\Gamma_{q, 1}$ and $\Gamma_{q, 2}$ (in $\mathrm{V} \mathrm{m}{ }^{2}$ ) are the intensities of the horizontal and vertical components of the $q$ th source dipole, 


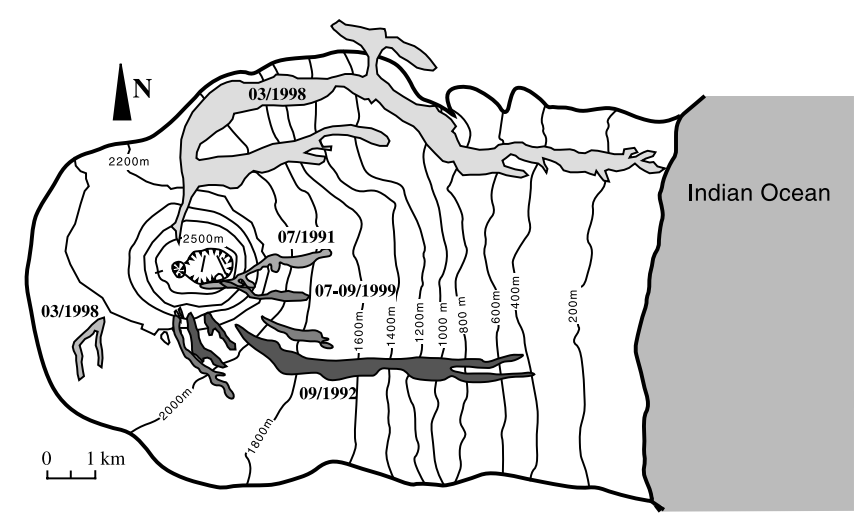

Figure 6. Map of the Piton de la Fournaise volcano (Réunion Island, Indian Ocean). During the period investigated (1991-1999), the main eruptive event is that of March 1998.

respectively. The two source terms entering equation (58) are defined by:

and

$$
\Gamma_{q, 1} \equiv \frac{p_{q} \sin \psi_{q}}{4 \pi \varepsilon}
$$

$$
\Gamma_{q, 2} \equiv \frac{p_{q} \cos \psi_{q}}{4 \pi \varepsilon}
$$

where $\Gamma_{q, 1}=0$ for a vertical dipole, $\psi_{q}=0, \pi$, whereas $\Gamma_{\mathrm{q}, 2}=0$ for a horizontal dipole, $\psi_{q}=\pi / 2,-\pi / 2$. The electrical field at point $P$ along the profile is given by:

$$
\begin{gathered}
E_{u}(P) \equiv-\frac{\partial \varphi(P)}{\partial u}=-\left[\frac{\partial \varphi}{\partial x}+\frac{\partial \varphi}{\partial z} \frac{d z}{d x}\right] \frac{d x}{d u}, \\
E_{u}(P)=\sum_{q=1}^{Q}\left(\Gamma_{q, 1} I_{u, 1}+\Gamma_{q, 2} I_{u, 2}\right),
\end{gathered}
$$

where $u$ is the curvilinear coordinate of point $P$ along the self-potential profile describing the ground surface, $d z / d x$ is the slope effect of the curvilinear elevation profile describing the ground surface, and the two scanning functions $I_{u, 1}$ and $I_{u, 2}$ (in the terminology used by Patella [1997]) are:

$$
\begin{aligned}
& I_{u, 1}\left(x-x_{q}, z(x)-z_{q}\right)= \\
& \quad \cdot \frac{\left[-2\left(x-x_{q}\right)^{2}+\left(z-z_{q}\right)^{2}-3(d z / d x)\left(x-x_{q}\right)\left(z-z_{q}\right)\right]}{\left[\left(x-x_{q}\right)^{2}+\left(z-z_{q}\right)^{2}\right]^{5 / 2}}\left(\frac{d x}{d u}\right)
\end{aligned}
$$

and

$$
\begin{aligned}
& I_{u, 2}\left(x-x_{q}, z(x)-z_{q}\right)= \\
& \cdot \frac{\left[-3\left(x-x_{q}\right)\left(z-z_{q}\right)+(d z / d x)\left[-2\left(z-z_{q}\right)^{2}+\left(x-x_{q}\right)^{2}\right]\right]}{\left[\left(x-x_{q}\right)^{2}+\left(z-z_{q}\right)^{2}\right]^{5 / 2}}\left(\frac{d x}{d u}\right) .
\end{aligned}
$$

For a flat ground surface, the scanning functions are directly obtained by taking $d x / d u=1$ and $d z / d x=0$ in equations (63) and (64). The 2-D tomography is based on a crosscorrelation algorithm between the theoretical scanning functions $I_{u, 1}$ and $I_{u, 2}$ and the electrical self-potential field $E_{u}$ recorded at the ground surface in order to determine the most probable discretized dipole distribution at depth responsible for the self-potential anomaly recorded at the ground surface. A horizontal dipole occurrence probability (HDOP) $\eta_{1}$ and a vertical dipole occurrence probability (VDOP) $\eta_{2}$ are determined for such a purpose,

$$
\begin{aligned}
& \eta_{1}\left(x_{q}, z_{q}\right)=C_{1} \int_{-\infty}^{+\infty} E_{u}[x, z(x)] \cdot I_{u, 1}\left[x-x_{q}, z(x)-z_{q}\right] d x, \\
& \eta_{2}\left(x_{q}, z_{q}\right)=C_{2} \int_{-\infty}^{+\infty} E_{u}[x, z(x)] \cdot I_{u, 2}\left[x-x_{q}, z(x)-z_{q}\right] d x, \quad(66) \\
& C_{1} \equiv\left[\int_{-\infty}^{+\infty} E_{u}^{2}[x, z(x)] d x \int_{-\infty}^{+\infty} I_{u, 1}^{2}\left[x-x_{q}, z(x)-z_{q}\right] d x\right]^{-1 / 2} \\
& C_{2} \equiv\left[\int_{-\infty}^{+\infty} E_{u}^{2}[x, z(x)] d x \int_{-\infty}^{+\infty} I_{u, 2}^{2}\left[x-x_{q}, z(x)-z_{q}\right] d x\right]^{-1 / 2}
\end{aligned}
$$

where $C_{1}$ and $C_{2}$ are normalization factors, which include a normalization by the total power of the electrical field recorded at the ground surface. The integrals in equations (65)-(68) can be numerically evaluated since they are only related to the electrical field at the ground surface, which can be computed directly from the self-potential profile, and the scanning functions given by equations (63) and (64). The Cauchy-Schwarz inequality yields:

$$
\begin{aligned}
& \left(\int_{-\infty}^{+\infty} E_{u}[x, z(x)] I_{u, 1}\left[x-x_{q}, z(x)-z_{q}\right] d x\right)^{2} \leq \int_{-\infty}^{+\infty} E_{u}^{2}[x, z(x)] d x \\
& \quad \int_{-\infty}^{+\infty} I_{u, 1}^{2}\left[x-x_{q}, z(x)-z_{q}\right] d x \\
& \left(\int_{-\infty}^{+\infty} E_{u}[x, z(x)] I_{u, 2}\left[x-x_{q}, z(x)-z_{q}\right] d x\right)^{2} \leq \int_{-\infty}^{+\infty} E_{u}^{2}[x, z(x)] d x
\end{aligned}
$$

$$
\int_{-\infty}^{+\infty} I_{u, 2}^{2}\left[x-x_{q}, z(x)-z_{q}\right] d x
$$

[35] This yields $-1 \leq \eta_{1}\left(x_{q}, z_{q}\right) \leq 1$ and $-1 \leq \eta_{2}\left(x_{q}, z_{q}\right) \leq$ 1 using equations $(65)-(70)$. As $\operatorname{sign}\left[\eta_{1}\left(x_{q}, z_{q}\right)\right]=\operatorname{sign}\left(\Gamma_{q, 1}\right)$, 


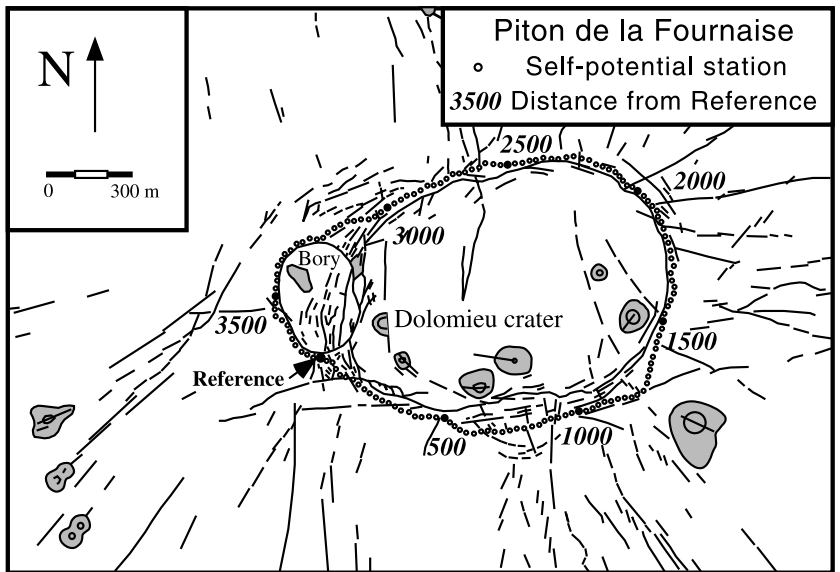

Figure 7. Map of the Bory and Dolomieu craters of the Piton de la Fournaise volcano. The measurement profile surrounds these craters. The open circles indicate the position of the self-potential stations. The measurements are carried out in the direction opposite to clockwise (see the distances in meters from the reference station). The pattern of surface fractures is taken from the work of Lénat and Bachèlery [1990]. The vents are in gray. the case $\eta_{1}>0$ corresponds to a dipole contribution oriented in the right side $\left(\psi_{q} \in[0, \pi]\right)$ according to the convention taken in Figure $3, \eta_{1}$ corresponds to a dipole contribution oriented in the left side $\left(\psi_{q} \in[-\pi, 0]\right)$. As $\operatorname{sign}\left[\eta_{2}\left(x_{q}, z_{q}\right)\right]=$ $\operatorname{sign}\left(\Gamma_{q, 2}\right)$, the case $\eta_{2}>0$ corresponds to a dipole contribution oriented upward $\left(\psi_{q} \in[-\pi / 2, \pi / 2]\right)$ and $\eta_{2}<0$ corresponds to a dipole contribution oriented downward $\left(\psi_{q}>\pi / 2\right.$ or $\left.\psi_{q}<-\pi / 2\right)$. In addition, a dipole occurrence probability (DOP) function and a phase angle are defined by:

$$
\begin{gathered}
\eta\left(x_{q}, z_{q}\right)=\sqrt{\eta_{1}^{2}\left(x_{q}, z_{q}\right)+\eta_{2}^{2}\left(x_{q}, z_{q}\right)}, \\
\theta\left(x_{q}, z_{q}\right)=\arctan \left[\frac{\eta_{1}\left(x_{q}, z_{q}\right)}{\eta_{2}\left(x_{q}, z_{q}\right)}\right],
\end{gathered}
$$

with the property $0 \leq \eta\left(x_{q}, z_{q}\right) \leq 1$. The DOP function represents the probability of finding in a point $\left(x_{q}, z_{q}\right)$ of the subspace $\Omega$ a dipole responsible for the self-potential anomaly observed at the ground surface. The subsurface is discretized and each element is scanned using discretized version of equation (65) to equation (66) to determine the values of the HDOP, VDOP, and DOP functions attached to

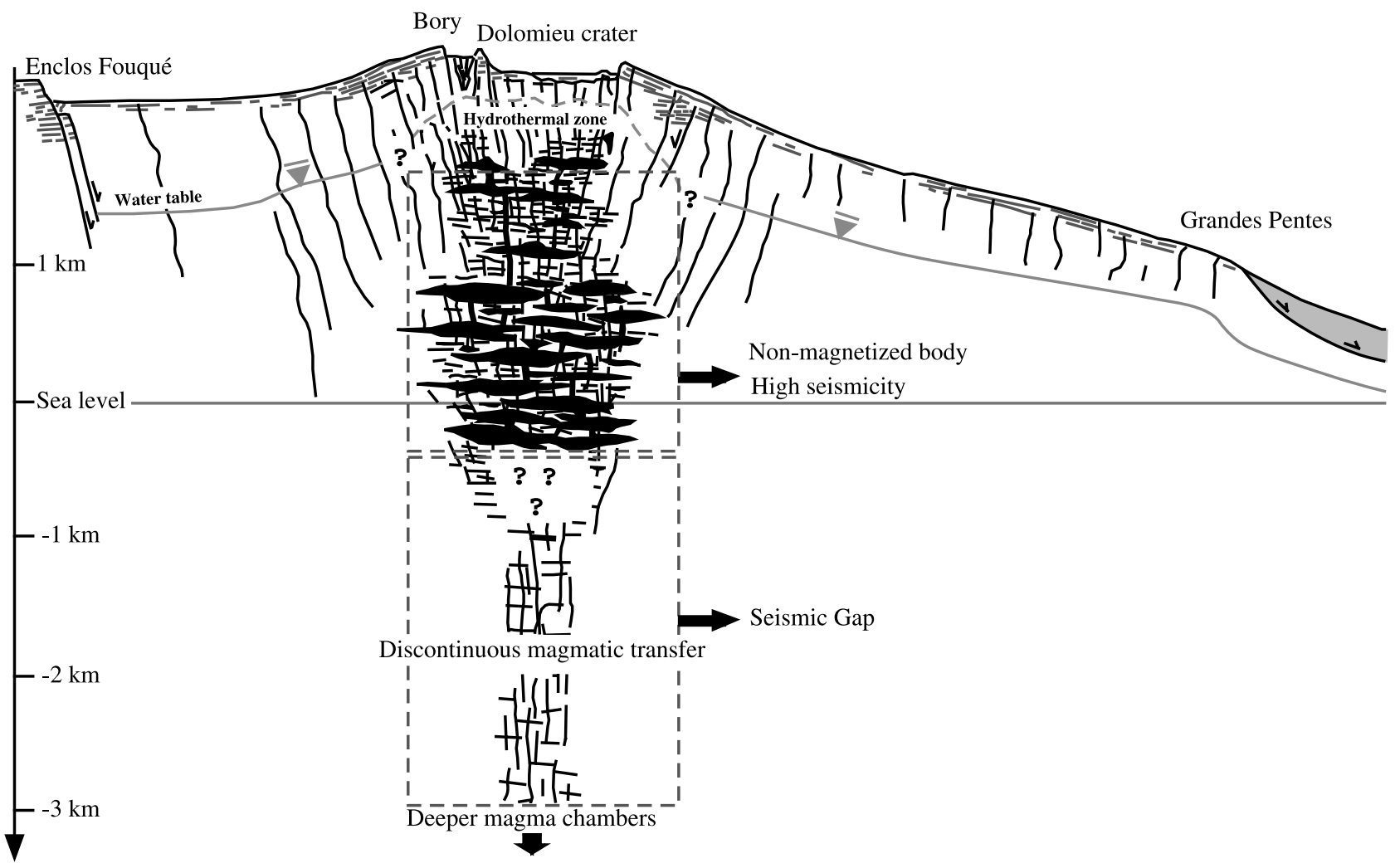

Figure 8. Sketch of the internal structure of the Piton de la Fournaise volcano (modified from the work of Lénat and Bachèlery [1990]). According to Lénat and Bachèlery [1990], the domain of magma storage is represented as a network of sills and dikes. The low seismicity observed inside the volcano below the sea level could be explained by either the bottom of the magma reservoir system or a ductile root with a higher percentage of magma. 
this element from the self-potential data recorded at the ground surface. Then these values are contoured to provide a tomographic image of the probable location of the dipole occurrence in the subsurface. The dipole occurrence probability $\eta\left(x_{q}, z_{q}\right)$ provides a tool for source recognition associated with dipolar polarization at depth.

\section{Field Case}

[36] In this section, we investigate self-potential disturbances associated with the preparation of a volcanic eruption at the Piton de la Fournaise volcano, a highly active basaltic shield volcano located in the southeastern part of the Réunion island (Figure 6). The major features of this volcano are a 400-m-high central cone, located inside the Enclos Fouqué depression, two diffuse curved rift zones that join together on the summit area, and two coalescent summit craters, Bory to the west and Dolomieu to the east (Figures 6-8). Historical activity has been mostly restricted to effusive eruptions on the central cone and along the rift zones. On the basis of geologic and geophysical data, Lénat and Bachelery [1990] inferred the presence of a shallow magma reservoir beneath the central cone (Figure 8). This reservoir appears to be composed of a complex of small magma bodies lying between $\sim 1$ and $2.5 \mathrm{~km}$ beneath the surface. The lateral extent of this storage zone is inferred by Lénat and Bachèlery [1990] to correspond approximately to that of the two summit craters. Self-potential data [Malengreau et al., 1994] and electromagnetic data [Lénat et al., 2000] strongly support the assumption of a well-developed hydrothermal system above the postulated magma reservoir, as previously suggested by Bachèlery and Lénat [1993].

\subsection{Field Measurement Procedures}

[37] Four different self-potential surveys have been conducted at the Piton de la Fournaise volcano between 1993 and 1999 along the same profile (see Figures 7 and 9). This profile forms a close loop (length $3.75 \mathrm{~km}$ and radius $\sim 600$ $\mathrm{m}$, which will be considered as the depth of investigation of tomography). Measurements are regularly spaced with an interval equal to $25 \mathrm{~m}$. These field investigations complete a first study made by Malengreau et al. [1994] during the period 1981-1992.

[38] Self-potential measurements are performed with two nonpolarizable electrodes, which comprise a copper rod immersed in a saturated copper sulphate $\left(\mathrm{CuSO}_{4}\right)$ solution at saturation. The microporous nature of the end-contacts of the electrodes (made in a low-permeability wood) avoids leakage of the $\mathrm{CuSO}_{4}$ solution during contact between with the ground. We use a high-impedance millivoltmeter $\left(\sim 10^{8}\right.$ $\Omega$ internal impedance) to carry out the measurements in the field. Before each series of measurements, the electrodes are put side by side in the same hole to check that the difference of potential is less than $2 \mathrm{mV}$.

[39] The measurements are made using one of the two electrodes as a fixed reference station. The second electrode is used to scan the electrical potential at the ground surface along the profile. At each measurement station, we dig a small hole (few centimeters deep). The moisture in the soil is most of the time sufficiently high to make the impedance contact between the electrode and the ground low enough to get good measurements. However, if the contact impedance
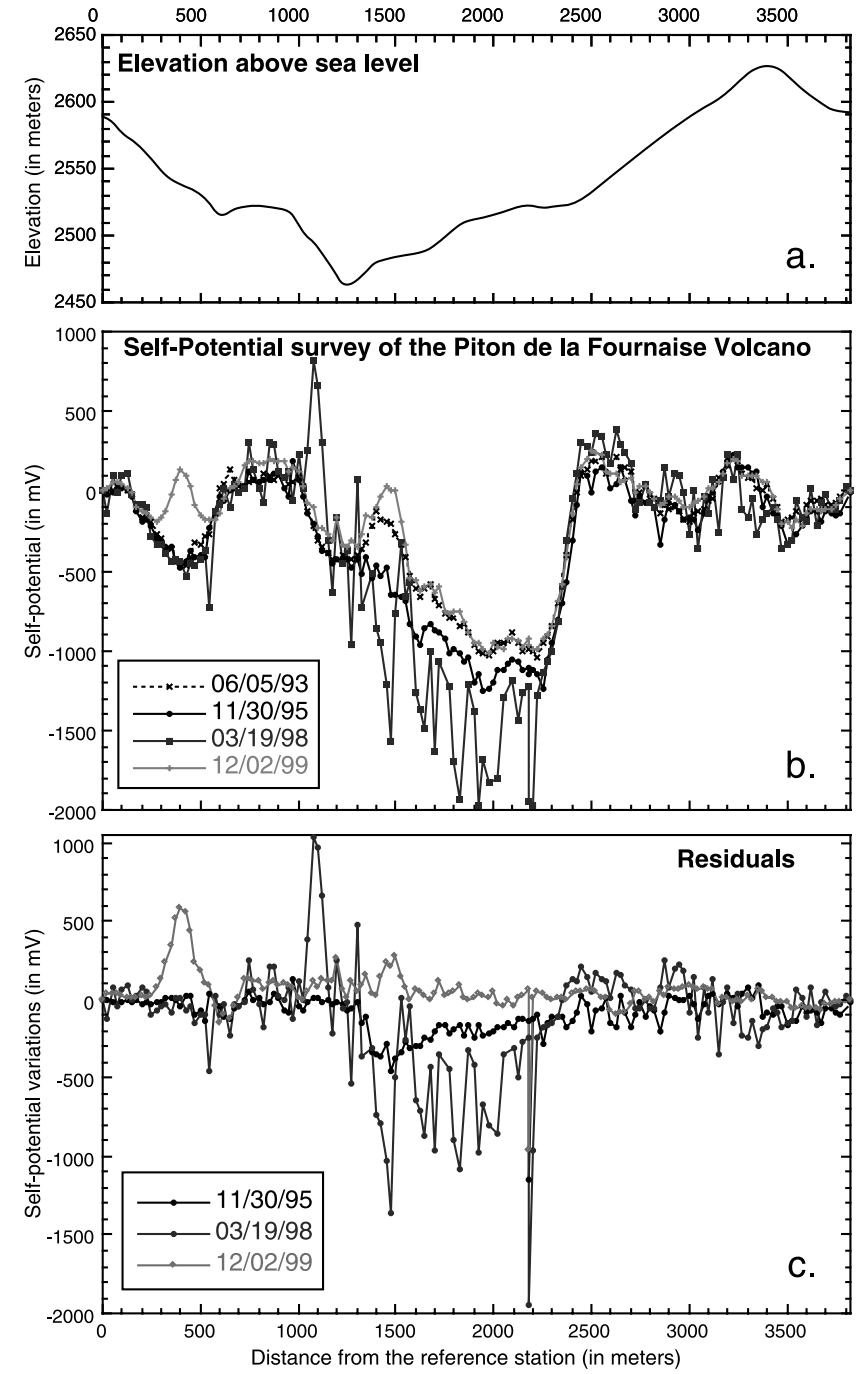

Figure 9. Self-potential profiles around the central active portion of the Piton de La Fournaise volcano. (a) Topography of the profile. (b) Self-potential signals preceding and following the March 1998 event and during the period 1993-1999. (c) Self-potential changes during the period 1993-1999 with a reference state taken on 5 June 1993. Note the electrical field change preceding the March 1998 eruption in the central portion of the profile. After the eruption, the signal comes back to the initial baseline given by the 1993 profile except at the beginning of the profile where a new $500 \mathrm{mV}$ amplitude anomaly develops.

is high $\left(>1 \mathrm{M} \Omega\right.$ ), a small amount of a saturated $\mathrm{CuSO}_{4}$ solution is placed at the bottom of the hole to improve the contact impedance. A long wire is used to connect the two electrodes. The distance between two successive measurements is $25 \mathrm{~m}$. The total length of the wire is $700 \mathrm{~m}$ in the present case, and consequently, a total of 28 measurements can be performed with the same reference. The advantage of this procedure is to avoid cumulative errors by changing the reference too often along the same profile. Every $700 \mathrm{~m}$, a new reference station is settled. As the profile is $3.75 \mathrm{~km}$ long, this means that $\sim 5$ reference base stations are used to obtain the entire profile around the summit craters. At the end of the profile, the entire self-potential profile is recon- 

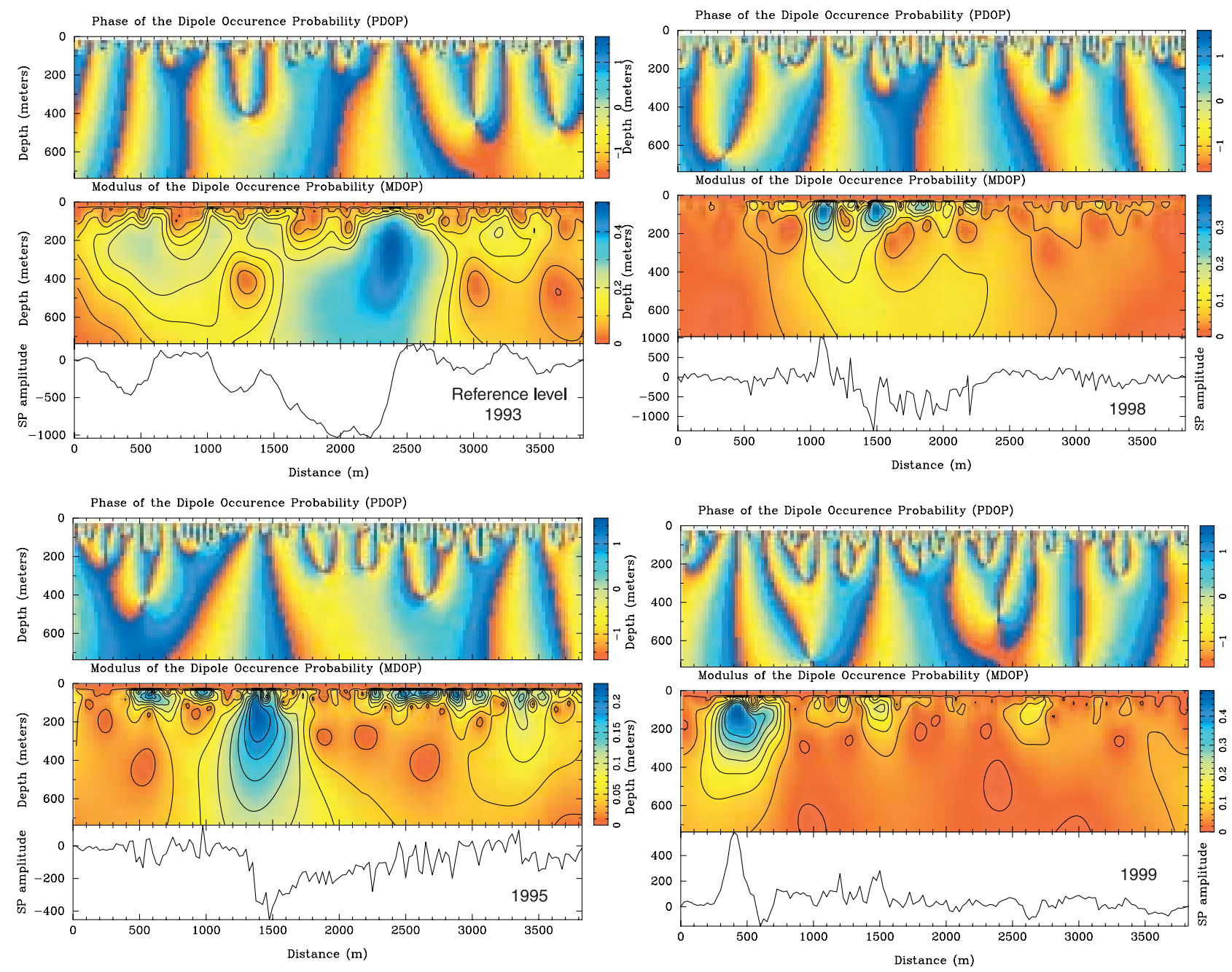

Figure 10. Dipolar tomography of the residual self-potential profiles. The first self-potential profile (1993 profile) is used as the reference. Tomography of the self-potential residues provides the depth locations of the dipolar sources responsible for self-potential changes since 1993.

structed using the first reference station as the unique reference for the entire profile. The quality of the measurements can be checked using the fact that the sum of the potential drops should go to zero along a close loop outside the source volume (Kirchoff's law). This is nearly the case here and the small residual voltage difference (few tens of millivolts) is redistributed over the entire profile to close effectively the loop.

\subsection{Data Analysis}

[40] During the period investigated, two main eruptions occurred. The first eruption occurred from August to September 1992, just few months prior the realization of the 1993 profile. The second main eruption occurred in March 1998. The self-potential profiles and the topography are shown in Figure 9. In Figure 10, we have plotted the self-potential changes using the 1993 profile as reference. They are several interesting features appearing on these profiles. In the preparation phase of the March 1998 eruption, several self-potential signal variations appear with a strength of 1-300 $\mathrm{mV}$. These disturbances are located in the central portion of the profile, in a place where volcanic activity is high (Figure 6). They are strong self-potential variations associated with the March 1998 eruption itself. After this eruption (see the 1999 profile in Figure 9), most of the self-potential signal comes back to the initial baseline of 1993 (except a marked anomaly in the first part of the profile).

[41] Dipolar tomographies of the four self-potential profiles are shown in Figure 10 using the 1993 profile as reference. This allows mapping the position of the sources responsible for self-potential changes since the reference state of 1993. We believe that these signals represent manifestation of groundwater flow associated with THM perturbations of the volcano. The negative anomaly implies rather a downward migration of the groundwater like associated with a fracturation dilatant zone (type I with dilatant zone). However, there is no enough data here to characterize the velocity of migration of the THM disturbances. Only future works with different geophysical tools involved at the same time will clarify the processes at work there. For example, fracturation processes associated with the propagation of shock waves can generate acoustic signals, which can be recorded at the ground surface. Can the concomitant record of electrical and seismic signals be used to bring 
complementary lights upon these hydro-mechanical processes is still an open question.

\section{3. $\quad$ Future Monitoring Operations}

[42] The obvious extension of the present work concerns continuous monitoring of the self-potential pattern at the top surface of a potentially dangerous volcano. During selfpotential monitoring operations, the main possible measurement errors arise from (1) electrode instability due to aging or chemical contamination and (2) telluric currents. The $\mathrm{Pb} / \mathrm{PbCl}_{2}$ electrodes designed by Petiau [2000] are extremely stable even to perform monitoring over several years [Trique et al., 1999]. Telluric currents result from temporal variations of the Earth's magnetic field, which induce an electrical current density in the ground. The fluctuation of the induced electrical field can range in duration from few milliseconds to decades and can produce electrical field gradients of several tens to hundreds of millivolts per kilometer over highly resistive areas. Therefore the monitoring of the self-potential field over a long period of time and over an extended area needs corrections for telluric currents. This can be accomplished, for example, by filtering the measurements using the magnetic variations recorded with a magnetometer and a reference station for the selfpotential survey. Kawakami and Takasugi [1994] showed this method can reduce the noise by order of magnitude.

\section{Concluding Statements}

[43] Substantial electrical disturbances are associated with THM disturbances in thermoporoelastic media. These electrical disturbances diffuse in a quasi-instantaneous fashion and bring information at the ground surface relative to the position and strength of the THM source in the system. The coupling between the THM disturbances and the electromagnetic fields they generate is electrokinetic in nature, i.e., due to a relative displacement between the mineral grains and the pore water. The coupling coefficient entering into this hydro-electric problem is measured in the laboratory for crushed basaltic and volcaniclastic rocks and scoria. Experimental results show that this electrokinetic coupling coefficient is of considerable magnitude, especially at near neutral $\mathrm{pH}$ and lowly mineralized water. Detectable electrical surges have been observed around the central portion of the Piton de la Fournaise volcano (Réunion island). These electrical field disturbances seem to be associated with the preparation of the March 1998 eruption of this volcano. The measurements of these self-potential signals could complement seismic monitoring networks in the purpose of volcanic activity forecasting.

\section{Appendix A: Thermoporoelastic Theory}

[44] We start by introducing the various parameters entering into the thermoporoelastic theory. Let us start by the conservation of fluid mass in a deforming porous medium, which is determined by [e.g., Palciauskas and Domenico, 1989]:

$$
\nabla \cdot\left(\rho_{f} \mathbf{u}\right)=\rho_{f}\left[-\frac{1}{V} \frac{d V}{d t}-\frac{1-\phi}{\rho_{S}} \frac{d \rho_{S}}{d t}-\frac{\phi}{\rho_{f}} \frac{d \rho_{f}}{d t}\right]
$$

where $\mathbf{u}$ represents the Darcy filtration velocity, $d($. represents here a Lagrangian derivative in a Lagrangian framework associated with a fixed amount of solid matrix, $V$ is the volume of an infinitesimal element of the porous rock, $t$ is the time, $\rho=\rho_{f} \phi+\rho_{S}(1-\phi)$ is the bulk density of the porous aggregate, $\phi$ is the porosity, and the subscripts $f$ and $S$ refer to the fluid and solid phases, respectively. The two last terms of the conservation of fluid mass are given by two constitutive relationships for the grain framework (the matrix) and the pore fluid,

$$
\begin{gathered}
(1-\phi) \frac{d \rho_{S}}{\rho_{S}}=\beta_{S} d \sigma-\phi \beta_{S} d p-(1-\phi) \alpha_{S} d T, \\
\frac{d \rho_{f}}{\rho_{f}}=\beta_{f} d p-\alpha_{f} d T, \\
\beta_{S} \equiv \frac{1-\phi}{\rho_{S}}\left(\frac{\partial \rho_{S}}{\partial \sigma}\right)_{p, T}, \\
\beta_{S^{\prime}} \equiv-\frac{1-\phi}{\phi \rho_{S}}\left(\frac{\partial \rho_{S}}{\partial p}\right)_{\sigma, T}, \\
\alpha_{S} \equiv \frac{-1}{\rho_{S}}\left(\frac{\partial \rho_{S}}{\partial T}\right)_{p, \sigma}, \\
\alpha_{f} \equiv-\frac{1}{\rho_{f}}\left(\frac{\partial \rho_{f}}{\partial T}\right)_{p}, \\
\beta_{f} \equiv \frac{1}{\rho_{f}}\left(\frac{\partial \rho_{f}}{\partial p}\right)_{T},
\end{gathered}
$$

where $\sigma=\left(\sigma_{11}+\sigma_{22}+\sigma_{33}\right) / 3$ is the mean confining stress, $\beta_{S}=1 / K_{S}$ and $\beta_{S^{\prime}}$ are two independent isothermal grain compressibilities (in $\mathrm{Pa}^{-1}$ ), $\alpha_{S}$ is the isobaric thermal expansion coefficient of the matrix (in ${ }^{\circ} \mathrm{C}^{-1}$ ), $\beta_{f}$ is the isothermal fluid compressibility (in $\mathrm{Pa}^{-1}$ ), and $\alpha_{f}$ is the isobaric thermal expansion coefficient for fluid (in ${ }^{\circ} \mathrm{C}^{-1}$ ). In a linear thermoporoelastic formulation, the effective stress tensor $\sigma_{i j}$ is given by [e.g., McTigue, 1986],

$$
\sigma_{i j}=\left(K-\frac{2}{3} \mu\right) \delta_{i j}+2 \mu \varepsilon_{i j}-\xi p-K \alpha_{b}\left(T-T_{0}\right) \delta_{i j},
$$

$$
\begin{gathered}
\xi \equiv 1-\frac{\beta_{S}}{\beta}, \\
\beta \equiv \frac{1}{K} \equiv \frac{1}{V}\left(\frac{\partial V}{\partial \sigma}\right)_{p, T},
\end{gathered}
$$

$$
\alpha_{b} \equiv(1-\phi) \alpha_{S}+\phi \alpha_{\phi},
$$


where $K$ and $\mu$ are the bulk and shear moduli of the porous material, respectively, $\zeta$ is one of the Biot coefficients, $\alpha_{b}$ is the isobaric thermal expansion coefficient of the porous material (in ${ }^{\circ} \mathrm{C}^{-1}$ ), $\alpha_{\phi}$ is the thermal expansion coefficient for the connected pores (in ${ }^{\circ} \mathrm{C}^{-1}$ ) and $\delta_{\mathrm{ij}}$ is the Kronecker delta. The porous material is characterized, at least, by eight independent parameters (for example, $\beta, \beta_{S}, \beta_{f}, \beta_{S^{\prime}}, \mu$, $\left.\alpha_{f}, \alpha_{S}, \alpha_{b}\right)$. Neglecting inertial terms and volumetric forces, the force balance equation is given by $\sigma_{i j, j}=0$. The bulk deformation and the deformation rate (in $\mathrm{s}^{-1}$ ) are given by:

$$
\begin{gathered}
\varepsilon=-\beta \sigma+\frac{p}{H}+\alpha_{b}\left(T-T_{0}\right), \\
\frac{d \varepsilon}{d t} \equiv \frac{1}{V} \frac{d V}{d t}=-\beta \frac{d \sigma}{d t}+\frac{1}{H} \frac{d p}{d t}+\alpha_{b} \frac{d T}{d t}, \\
\frac{1}{H} \equiv \frac{1}{\rho_{f}}\left(\frac{\partial m_{f}}{\partial \sigma}\right)_{p, T}=\beta-\beta_{S},
\end{gathered}
$$

where $1 / H$ (one of the Biot coefficients) represents a measure of the change in water content for a given change in confining stress when the material is permitted to drain freely. Combining the three constitutive relationships with the conservation of mass fluid gives two alternative equations for the conservation of fluid mass:

$$
\begin{gathered}
\nabla \cdot\left(\rho_{f} \mathbf{u}\right)=\rho_{f}\left[\frac{1}{H} \frac{d \sigma}{d t}+\alpha_{m} \frac{d T}{d t}-\frac{1}{R} \frac{d p}{d t}\right], \\
\nabla \cdot\left(\rho_{f} \mathbf{u}\right)=\rho_{f}\left[-\xi \frac{d \varepsilon}{d t}+\left(\alpha_{b} \xi+\alpha_{m}\right) \frac{d T}{d t}-\frac{1}{Q} \frac{d p}{d t}\right], \\
\frac{1}{R} \equiv \frac{1}{\rho_{f}}\left(\frac{\partial m_{f}}{\partial p}\right)_{\sigma, T}=\beta-\beta_{S}-\phi\left(\beta_{S^{\prime}}-\beta_{f}\right), \\
\frac{1}{Q} \equiv \frac{1}{\rho_{f}}\left(\frac{\partial m_{f}}{\partial p}\right)_{\varepsilon, T}=\frac{1}{R}-\frac{\xi}{H}, \\
\alpha_{m} \equiv-\frac{1}{\rho_{f}}\left(\frac{\partial m_{f}}{\partial T}\right)_{\sigma, p}=\phi\left(\alpha_{f}-\alpha_{\phi}\right) .
\end{gathered}
$$

The coefficient $1 / R$ represents a measure of the change in water content for a given change in pore fluid pressure when the porous material is permitted to drain freely, $1 / Q$ represents a measure of the amount of water which can be forced into a porous material under pressure while the volume of the material is kept constant, and $\alpha_{m}$ (in ${ }^{\circ} \mathrm{C}^{-1}$ ) describes how much fluid mass of water is expelled out of the porous material when the temperature is increased by $\theta \equiv\left(T-T_{0}\right)$. The main fluid pressure source associated with a magmatic intrusion in depth is the rapid increase of the temperature in the surrounding porous rock. This can be easily been shown from equations (A16) and (A17).
Indeed, the pore fluid pressure change with temperature in undrained conditions is given by:

$$
\left(\frac{\partial p}{\partial T}\right)_{\sigma, m_{f}} \leq\left(\frac{\partial p}{\partial T}\right) \leq\left(\frac{\partial p}{\partial T}\right)_{\varepsilon, m_{f}}
$$

and

$$
R \alpha_{m} \leq\left(\frac{\partial p}{\partial T}\right) \leq Q\left(\alpha_{b} \xi+\alpha_{m}\right)
$$

Basaltic and mudstone-type of materials would form the two end-members (hard and soft) which are generally forming the volcanic edifice. Palciauskas and Domenico [1989] give $R \alpha_{m}=0.88$ and $0.18 \mathrm{MPa}{ }^{\circ} \mathrm{C}^{-1}$ for a typical basalt and mudstone, respectively, and $Q\left(\alpha_{b} \xi+\alpha_{m}\right)=1.03$ and 1.14 $\mathrm{MPa}{ }^{\circ} \mathrm{C}^{-1}$, respectively. A magmatic intrusion can heat the surrounding porous rock at temperatures $>100^{\circ}$ above the background temperature. This can increase the pore fluid pressure to such levels that the rock surrounding the magmatic intrusion can be completely hydrofractured [e.g., Natale et al., 1998]. Equation (A16) combined with the Darcy equation yields equation (6) of the main text.

[45] The distribution of the temperature $T$ in the porous medium obeys the energy conservation law. The Fourier's law (including a convective heat transport term) and the heat energy conservation (neglecting the fluid-rock friction) are given by:

$$
\begin{aligned}
& \mathbf{h}=-\lambda \nabla T+\rho_{f} c_{f} T \mathbf{u}, \\
& \nabla \cdot \mathbf{h}=-\rho c \frac{\partial T}{\partial t}+Q,
\end{aligned}
$$

respectively, where $\mathbf{h}$ is the heat flux (in $\mathrm{J} \mathrm{m}^{-2} \mathrm{~s}^{-1}$ ), $\rho$ and $c$ are the bulk density (in $\mathrm{kg} \mathrm{m}^{-3}$ ) and the specific heat (in $\mathrm{J}$ $\mathrm{kg}^{-1}{ }^{\circ} \mathrm{C}^{-1}$ ) of the porous medium, respectively, $\rho_{f}$ and $c_{f}$ are the bulk density (in $\mathrm{kg} \mathrm{m}^{-3}$ ) and the specific heat (in $\mathrm{J} \mathrm{kg}^{-1}$ ${ }^{\circ} \mathrm{C}^{-1}$ ) of the pore water, respectively, and $Q$ is the bulk rate of heat production in the matrix (in $\mathrm{J} \mathrm{m}^{-3} \mathrm{~s}^{-1}$ ), which will be neglected below. Combining equations (A23) and (A24) leads to the following result:

$$
-\lambda \nabla^{2} T+\rho_{f} c_{f}(\mathbf{u} \cdot \nabla T+T \nabla \cdot \mathbf{u})=-\rho c \frac{\partial T}{\partial t},
$$

$$
\frac{\partial T}{\partial t}-\eta_{T} \nabla^{2} T-\beta_{T} \nabla p \cdot \nabla T=0
$$

where $\eta_{T}$ is the thermal diffusivity of the porous material (in $\mathrm{m}^{2} \mathrm{~s}^{-1}$ ) and $\beta_{T}$ (in $\mathrm{m}^{2} \mathrm{~Pa}^{-1} \mathrm{~s}^{-1}$ ) is the convective thermal diffusivity defined by:

$$
\eta_{T} \equiv \frac{\lambda}{\rho c}
$$

and

$$
\beta_{T} \equiv \frac{\rho_{f} c_{f} k}{\rho c \eta_{f}} .
$$


In these equations $\lambda$ is the thermal conductivity of the porous medium (in $\mathrm{W} \mathrm{m}^{-1}{ }^{\circ} \mathrm{C}^{-1}$ ), and $c_{f}$ the specific heat of the convecting fluid (in $\mathrm{J} \mathrm{m}^{-3}{ }^{\circ} \mathrm{C}^{-1}$ ). We note $\tau$ as the characteristic time for a temperature disturbance to move through conduction between two points separated by the distance $L$. We have $\tau=L^{2} / \eta_{T}$. Taking $L=1000 \mathrm{~m}, \eta_{T}=$ $10^{-6} \mathrm{~m}^{2} \mathrm{~s}^{-1}$, we obtain $\tau=30,000$ days. Consequently, transmission of the thermal information by conduction is an extremely slow process. Upsurge of pressure/temperature nonlinear waves (type II) is much more efficient to displace heat (and energy) in the system away from the source after the formation of an initial thermal shock.

\section{Appendix B: Electrokinetic Theory}

[46] We consider a water-saturated rock porous volume $\Omega$ isotropic and possibly inhomogeneous due to the presence of fractures or rock lithological discontinuities. When the pore water moves through the porous rock, electrical and hydraulic processes are coupled through the following macroscopic constitutive equations operating at the scale of a representative elementary volume of the porous material inside $\Omega$ [e.g., Ishido and Mizutani, 1981]:

$$
\begin{gathered}
\mathbf{j}=\sigma \mathbf{E}-L\left(\nabla p-\rho_{f} \mathbf{g}\right), \\
\mathbf{u}=L \mathbf{E}-\frac{k}{\eta_{f}}\left(\nabla p-\rho_{f} \mathbf{g}\right), \\
L=-\frac{\varepsilon_{f} \zeta}{\eta_{f} F}, \\
C \equiv\left(\frac{\partial \varphi}{\partial p}\right)_{\mathbf{j}=0}=-L / \sigma
\end{gathered}
$$

where $\mathbf{j}$ is the electrical current density (in $\mathrm{A} \mathrm{m}^{-2}$ ), $\mathbf{u}$ is the volumetric fluid flux (in $\mathrm{m} \mathrm{s}^{-1}$ ) (Darcy velocity), $\mathbf{E}$ is the electrical field (in $\mathrm{V} \mathrm{m}^{-1}$ ), $p$ is the pore fluid pressure (in $\mathrm{Pa}$ ), $\mathbf{g}$ is the gravity acceleration vector (in $\mathrm{m} \mathrm{s}^{-2}$ ), $\sigma$ and $k$ are the electrical conductivity (in $\mathrm{S} \mathrm{m}^{-1}$ ) and intrinsic permeability (in $\mathrm{m}^{2}$ ) of the porous medium, respectively, $\varepsilon_{f}$ is the dielectric constant of the pore water (in $\mathrm{F} \mathrm{m}^{-1}$ ), $\eta_{f}$ is the dynamic shear viscosity of the pore water (in $\mathrm{Pa} \mathrm{s}$ ), and $L$ is the electrokinetic coupling term (in $\mathrm{m}^{2} \mathrm{~V}^{-1} \mathrm{~s}^{-1}$ ) between the Darcy and generalized Ohm laws, $C$ (in $\mathrm{V} \mathrm{Pa}^{-1}$ ) is the streaming potential coupling coefficient. The parameter $\zeta$ (in V) entering into the determination of the coupling coefficient $L$ is named the "zeta potential." It represents the electrical potential at the mineral/water interface. The term $F$ is the (dimensionless) electrical formation factor.

[47] Inspection of equations (B1) and (B2) show that the second equation can be safely decoupled from the first equation if the only component of the electrical field is that produced through the electrokinetic coupling [e.g., Revil et al., 1999, section 3.1]. So we recover the Darcy equation:

$$
\mathbf{u} \approx-\frac{k}{\eta_{f}}\left(\nabla p-\rho_{f} \mathbf{g}\right)
$$

[48] Now we look for a representation of the electrical field $\mathbf{E}$ at the observation station $P$. The polarization of charge inside the volcanic edifice is due to electrokinetic sources located within $\Omega$ bounded by the surface $\partial \Omega$. These electrokinetic sources are associated with fluid flow resulting from stress and thermal disturbances related to a magmatic intrusion at depth. The relationship between the electrical current density $\mathbf{j}$ and the Darcy velocity $\mathbf{u}$ is,

$$
\mathbf{j}=\sigma \mathbf{E}+\frac{\nu_{f} L}{k}\left(\rho_{f} \mathbf{u}\right),
$$

where $v_{f} \equiv \eta_{f} / \rho_{f}$ is the kinematic viscosity of the pore fluid. In the quasi-static limit,

$$
\begin{gathered}
\nabla \times \mathbf{E}=-\mu \frac{\partial \mathbf{H}}{\partial t} \approx 0, \\
\nabla \cdot \mathbf{j}=0,
\end{gathered}
$$

and therefore $\mathbf{E}=-\nabla \varphi$. The use of equations (B5)-(B8) yields:

$$
\begin{gathered}
\nabla \cdot(\sigma \mathbf{E})=-\frac{\nu_{f} L}{k} \nabla \cdot\left(\rho_{f} \mathbf{u}\right)-\rho_{f} \mathbf{u} \cdot \nabla\left(\frac{\nu_{f} L}{k}\right), \\
\sigma \nabla^{2} \varphi-\mathbf{E} \cdot \nabla \sigma=\frac{\nu_{f} L}{k} \nabla \cdot\left(\rho_{f} \mathbf{u}\right)+\rho_{f} \mathbf{u} \cdot \nabla\left(\frac{\nu_{f} L}{k}\right), \\
\nabla^{2} \varphi(\mathbf{r}, t)=-\frac{\nu_{f} C}{k} \nabla \cdot\left(\rho_{f} \mathbf{u}\right)+\frac{\rho_{f} \mathbf{u}}{\sigma} \cdot \nabla\left(\frac{\nu_{f} L}{k}\right)+\mathbf{E} \cdot \frac{\nabla \sigma}{\sigma} .
\end{gathered}
$$

[49] If we consider a volume $\Omega$ bounded by the surface $\partial \Omega$ (n outward normal unit vector to $\partial \Omega$ ), the integration of equation (B11) by the Green's method yields at observation station $P$ :

$$
\begin{gathered}
\varphi(\mathbf{r}, t)=\varphi_{1}(\mathbf{r}, t)+\varphi_{2}(\mathbf{r}, t)+\varphi_{3}(\mathbf{r}, t) \\
+\frac{1}{4 \pi} \int_{\partial \Omega}\left[\frac{\nabla \varphi}{x}-\varphi \nabla\left(\frac{1}{x}\right)\right] \cdot \mathbf{n} d S, \\
\varphi_{1}(\mathbf{r}, t)=\frac{-1}{4 \pi} \int_{\Omega} S \frac{\nabla \cdot\left[\rho_{f} \mathbf{u}\left(\mathbf{r}^{\prime}, t\right)\right]}{x} d V, \\
\varphi_{2}(\mathbf{r}, t)=\frac{1}{4 \pi} \int_{\Omega} \frac{\mathbf{E}\left(\mathbf{r}^{\prime}, t\right)}{x} \cdot \frac{\nabla \rho}{\rho} d V, \\
\varphi_{3}(\mathbf{r}, t)=\frac{-1}{4 \pi} \int_{\Omega} \nabla S \cdot\left(\frac{\rho_{f} \mathbf{u}\left(\mathbf{r}^{\prime}, t\right)}{x}\right) d V,
\end{gathered}
$$

$$
S \equiv \frac{C \eta_{f}}{\rho_{f} k},
$$


where $x \equiv\left|\mathbf{r}-\mathbf{r}^{\prime}\right|$ is the distance between $P$ and the infinitesimal volume element $d V$ of the conductive medium surrounding the integration point $M$ and $S$ (in $\mathrm{V} \mathrm{m} \mathrm{s} \mathrm{kg}^{-1}$ ) is the electrokinetic coupling term for the self-potential problem. The volume of integration $\Omega$ corresponds to the whole volume in which fluid flow takes place. The first contribution to the self-potential $\varphi_{1}(\mathbf{r}, t)$ represents the primary source term and the second contribution $\varphi_{2}(\mathbf{r}, t)$ represents the electrical potential related to charge accumulation at the heterogeneities of the porous volume (secondary source). The third term corresponds to charge accumulation at heterogeneities of the coupling term $S$. Far from the source volume, $\Omega$, the electrical field vanishes. If the surface $\partial \Omega$ corresponds to the boundary with an insulating medium (like the atmosphere), then $\mathbf{n} \cdot \nabla \varphi=0$, i.e., the normal component of the electrical current density vanishes on this surface, and the term $4 \pi$ in (B12) to (B15) needs to be replaced by $2 \pi$ (the sources of potential are contained in a half-space). Using equation (A16), the primary self-potential contribution is related to the stress and thermal disturbances by:

$$
\varphi_{1}(\mathbf{r}, t)=\frac{1}{4 \pi} \int_{\Omega} \frac{S \rho_{f}}{x}\left[\frac{1}{H} \frac{\partial \sigma}{\partial t}+\alpha_{m} \frac{\partial T}{\partial t}-\frac{1}{R} \frac{\partial p}{\partial t}\right] d V
$$

It results in two electrokinetic source terms associated with the variation with time of the confining stress and temperature, and a third term associated with the diffusive change of the pore fluid pressure with time. Therefore the observation of the time change of electrical signals recorded at the ground surface of an active volcano can evidence in situ changes in the local stress field.

[50] Acknowledgments. One of us, André Revil, thanks Pierre Choukroune and Bruno Hamelin for their support over several years at CEREGE, O. C. Clint for a copy of his Ph.D. thesis, and P. Henry, F. Perrier, and P. Gex for stimulating discussions. We thank the French National Research Council (CNRS) and the Ministère de la Recherche et de l'Education Nationale (MENRT, ACI-Jeune 0693, 1999 and ACI "Eau et Environnement 2001") for financial support. We thank T. Grosmann, M. Jaubert, B. Usclat, and D. Hermitte for help with the laboratory measurements. This work is dedicated to M. Aubert and P. Morat for their great contributions to the field of self-potential signals.

\section{References}

Bachèlery, P., and J.-F. Lénat, Le Piton de la Fournaise, Mem. Soc. Geol. Fr. 163, 221-229, 1993.

Bernabé, Y., Streaming potential in heterogeous networks, J. Geophys. Res., 103, 20,827-20,841, 1998.

Büttner, R., B. Zimanowski, and H. Röder, Short-time electrical effects during volcanic eruption: Experiments and field measurements, J. Geophys. Res., 105, 2819-2827, 2000.

Clint, O. C., Electrical potential changes and acoustic emissions generated by fracture and fluid flow during experimental triaxial rock deformation, Ph.D. thesis, 236 pp., Univ. Coll. London, London, 1999.

Del Negro, C., F. Ferrucci, and R. Napoli, Retrieval of large volcanomagnetic effects observed during the 1981 eruption of Mt. Etna, Ann. Geophys., 40, 547-562, 1997.

Di Maio, R., and D. Patella, Self-potential anomaly generation in volcanic areas. The Mont Etna case history, Acta Vulcanol., 4, 124-199, 1994.

Di Maio, R., V. Di Sevo, S. Giammetti, D. Patella, S. Piscitelli, and C. Silenziario, Self-potential anomalies in some Italian volcanic areas, Ann. Geophys., 39, 179-188, 1996.

Di Maio, R., P. Mauriello, D. Patella, Z. Petrillo, S. Piscitelli, and A. Siniscalchi, Electric and electromagnetic outline of the Mount SommaVesusius structural setting, J. Volcanol. Geotherm. Res., 82, 219-238, 1998.
Fitterman, D. V., Electrokinetic and magnetic anomalies associated wth dilatant regions in a layered Earth, J. Geophys. Res., 83, 5923-5928, 1978 .

Flekkøy, E. G., A. Malthe-Sørenssen, and B. Jamtveit, Modeling hydrofracture, J. Geophys. Res., 107(B8), doi:10.1029/2000JB000132, 2002.

Fontaine, F. J., M. Rabinowitcz, J. Boulègue, and L. Jouniaux, Constraints on hydrothermal processes on basaltic edifices: Inferences on the conditions leading to hydrovolcanic eruptions at Piton de la Fournaise, Réunion island, Indian Ocean, Earth Planet. Science Lett., 200, 1-14, 2002.

Fournier, C., Spontaneous potentials and resistivity surveys applied to hydrogeology in a volcanic area: Case history of the Chaîne des Puys (Puyde-Dôme, France), Geophys. Prospect., 37, 647-668, 1989.

Garcìa, R., and G. Natale, Subsurface propagation of thermomechanical kinks through hydrothermal fluid-saturated porous horizons, J. Geophys. Res., 104, 20,059-20,068, 1999.

Gibert, D., and M. Pessel, Identification of sources of potential fields with the continuous wavelet transform: Application to self-potential profiles, Geophys. Res. Lett., 28, 1863-1866, 2001.

Haartsen, M. W., W. Dong, and M. N. Toksöz, Dynamic streaming currents from seismic point sources in homogeneous poroelastic media, Geophys. J. Int., 132, 256-274, 1998.

Haimson, B. C., and F. Rummel, Hydrofracturing stress measurements in the Iceland research drilling project drill hole at Reydarfjordur, Iceland, J. Geophys. Res., 87, 6631-6649, 1982.

Hashimoto, T., and Y. Tanaka, A large self-potential anomaly on Unzen volcano, Shimabara peninsula, Kyushu island, Japan, Geophys. Res. Lett., 22, 193-194, 1995.

Ishido, T., and H. Mizutani, Experimental and theoretical basis of electrokinetic phenomena in rock-water systems and its application to geophysics, J. Geophys. Res., 86, 1763-1775, 1981.

Johnston, M. J. S., and F. D. Stacey, Volcanomagnetic effect observed on Mt Ruapehu, New Zealand, J. Geophys. Res., 74, 6541-6544, 1969.

Kawakami, N., and S. Takasugi, SP monitoring during the hydraulic fracturing using the TG-2 well, paper 1004 presented at EAEG 56th Meeting and Exhibition, Vienna, Austria, June 6-10, 1994.

Kostek, S., L. M. Schwartz, and D. L. Johnson, Fluid permeability in porous media: Comparison of electrical estimates with hydrodynamical calculations, Phys. Rev. B, 45, 186-195, 1992.

Lénat, J.-F., and P. Bachèlery, Structure et fonctionnement de la zone centrale du Piton de la Fournaise, in Le Volcanisme de la Réunion, Monographie (in French), edited by J.-F. Lénat, pp. 257-296, Centre de Recherches Volcanologiques, Clermont-Ferrand, France, 1990.

Lénat, J.-F., D. Fitterman, D. B. Jackson, and P. Labazuy, Geoelectrical structure of the central zone of Piton de la Fournaise volcano (Réunion), Bull. Volcanol., 62, 75-89, 2000.

Lorne, B., F. Perrier, and J.-P. Avouac, Streaming potential measurements, 1, Properties of the electrical double layer from crushed rock samples, J. Geophys. Res., 104, 17,857-17,877, 1999.

Malengreau, B., J.-F. Lénat, and A. Bonneville, Cartography and temporal observation of self-potential (SP) anomalies at Piton de la Fournaise (in French with extended English abstract), Bull. Soc. Geol. Fr., 165, $221-$ 232, 1994

Matsumoto, N., T. Sato, N. Matsushima, F. Akita, T. Shibata, and A. Suzuki, Hydrological anomalies associated with crustal deformation before the 2000 eruption of Usu volcano, Japan, Geophys. Res. Lett., 29(5), doi:10.1029/2001GL013968, 2002.

McTigue, D. F., Thermoelastic response of fluid-saturated rock, J. Geophys. Res., 91, 9533-9542, 1986.

Merlani, A. L., G. Natale, and E. Salusti, Fracturing processes due to temperature and pressure nonlinear waves propagating in fluid-saturated porous rocks, J. Geophys. Res., 106, 11,067-11,081, 2001.

Michel, S., and J. Zlotnicki, Self-potential and magnetic surveying of La Fournaise volcano (Réunion island): Correlations with faulting, fluid circulation, and eruption, J. Geophys. Res., 103, 17,845-17,857, 1998.

Natale, G., The effect of fluid thermal expansivity on thermo-mechanical solitary shock waves in the underground of volcanic domains, Pure Appl. Geophys., 152, 193-211, 1998.

Natale, G., E. Salusti, and A. Troisi, Rock deformation and fracturing processes due to nonlinear shock waves propagating in hyperthermal fluid pressurized domains, J. Geophys. Res., 103, 15,325-15,338, 1998.

Palciauskas, V. V., and P. A. Domenico, Fluid pressures in deforming porous rocks, Water Resour. Res., 25, 203-213, 1989.

Patella, D., Introduction to ground surface self-potential tomography, Geophys. Prospect., 45, 653-681, 1997.

Petiau, G., Second generation of lead-lead chloride electrodes for geophysical applications, Pure Appl. Geophys., 157, 357-382, 2000.

Poldini, E., Geophysical exploration by spontaneous polarization methods, Min. Mag., 59, 278-282, 347-352, 1938. 
Pride, S. R., Governing equations for the coupled electromagnetics and acoustics of porous media, Phys. Rev. B, 50, 15,678-15,696, 1994.

Revil, A., Comment on "Rapid fluid disruption: A source for self-potentia anomalies" by M. J. S. Johnston et al., J. Geophys. Res., 107(B8), doi:10.1029/2001JB000788, 2002a.

Revil, A., Genesis of mud volcanoes in sedimentary basins. A solitary wave-based mechanism, Geophys. Res. Lett., 29(12), doi:10.1029/ 2001GL014465, 2002b.

Revil, A., The hydroelectric problem of porous rocks: Thermodynamic approach and introduction of a percolation threshold, Geophys. J. Int., 151(3), 944-949, 2002c.

Revil, A., and P. Leroy, Hydroelectric coupling in a clayey material, Geophys. Res. Lett., 28, $1643-1646,2001$.

Revil, A., H. Schwaeger, L. M. Cathles, and P. D. Manhardt, Streaming potential in porous media, 2, Theory and application to geothermal systems, J. Geophys. Res., 104, 20,033-20,048, 1999.

Revil, A., L. Ehouarne, and E. Thyreault, Tomography of self-potential anomalies of electrochemical nature, Geophys. Res. Lett., 28, $4363-$ 4366, 2001.

Revil, A., D. Hermitte, M. Voltz, R. Moussa, J.-G. Lacas, G. Bourriè, and F. Trolard, Self-potential signals associated with variations of the hydraulic head during an infiltration experiment, Geophys. Res. Lett., 29(7), doi:10.1029/2001GL014294, 2002a.

Revil, A., D. Hermitte, E. Spangenberg, and J. J. Cochemé, Electrical properties of zeolitized volcaniclastic materials, J. Geophys. Res., 107(B8), doi:10.1029/2001JB000599, 2002b.
Sailhac, P., and G. Marquis, Analytic potentials for the forward and inverse modeling of SP anomalies caused by subsurface fluid flow, Geophys. Res. Lett., 28, 1851-1854, 2001.

Sasai, Y., et al., Volcanomagnetic effect observed during the 1986 eruption of Izu-Oshima volcano, J. Geomagn. Geoelectr., 42, 291-317, 1990.

Schultz, R. A., Limits of strength and deformation properties of jointed basaltic rock masses, Rock Mech. Rock Eng., 28, 1-15, 1995.

Tanaka, Y., Eruption mechanism as inferred from geomagnetic changes with special attention to the 1989-1990 activity of Aso volcano, J. Volcanol. Geotherm. Res., 56, 319-338, 1993.

Trique, M., P. Richon, F. Perrier, J. P. Avouac, and J. C. Sabroux, Radon emanation and electric potential variations associated with transient deformation near reservoir lakes, Nature, 399, 137-141, 1999.

Yoshida, S., Convection current generated prior to rupture in saturated rocks, J. Geophys. Res., 106, 2103-2120, 2001.

Zlotnicki, J., and J. L. Le Mouël, Volcanomagnetic effects observed on Piton de la Fournaise volcano (Réunion island): 1985-1987, J. Geophys. Res., 93, 9157-9171, 1988.

P. Labazuy, Laboratoire Magmas et Volcans, Université Blaise Pascal, 5 rue Kessler, 63038, Clermont-Ferrand, France.

A. Revil and G. Saracco, Department of Hydrogeophysics and Porous Media, CNRS-Centre Européen de Recherche et d'Enseignement des Géosciences de l'Environment (CNRS-CEREGE), BP 80, 13545 Aix-enProvence Cedex 04, France. (revil@cerege.fr) 